DRAFT VERSION MAY 1, 2019

Typeset using LATEX twocolumn style in AASTeX62

\title{
Composition Effects on Kilonova Spectra and Light Curves: I
}

\author{
Wesley Even, ${ }^{1,2,3,4}$ Oleg Korobkin, ${ }^{1,2,3}$ Christopher L. Fryer, ${ }^{1,2,3,5,6,7}$ Christopher J. Fontes, ${ }^{1,8}$ R. T. Wollaeger, ${ }^{1,3}$ \\ Aimee Hungerford, ${ }^{1,2,8}$ Jonas Lippuner, ${ }^{1,2,3}$ Jonah Miller, ${ }^{1,3,9}$ Matthew R. Mumpower, ${ }^{1,2,10}$ And \\ G. WENDELL MISCH${ }^{1,2,10}$

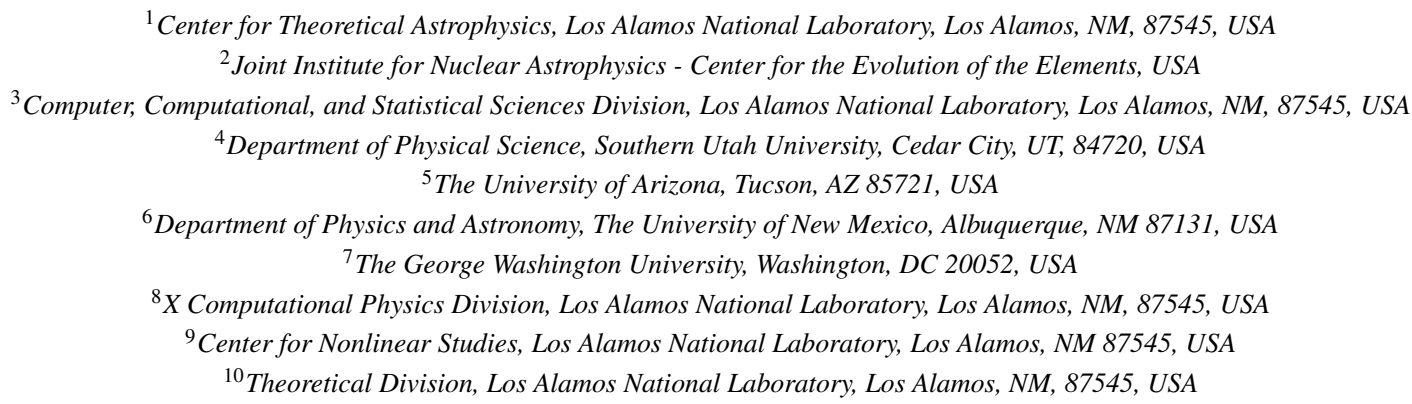

\section{ABSTRACT}

The merger of neutron star binaries is believed to eject a wide range of heavy elements into the universe. By observing the emission from this ejecta, scientists can probe the ejecta properties (mass, velocity and composition distributions). The emission (a.k.a. kilonova) is powered by the radioactive decay of the heavy isotopes produced in the merger and this emission is reprocessed by atomic opacities to optical and infra-red wavelengths. Understanding the ejecta properties requires calculating the dependence of this emission on these opacities. The strong lines in the optical and infra-red in lanthanide opacities have been shown to significantly alter the lightcurves and spectra in these wavelength bands, arguing that the emission in these wavelengths can probe the composition of this ejecta. Here we study variations in the kilonova emission by varying individual lanthanide (and the actinide uranium) concentrations in the ejecta. The broad forest of lanthanide lines makes it difficult to determine the exact fraction of individual lanthanides. $\mathrm{Nd}$ is an exception. Its opacities above 1 micron are higher than other lanthanides and observations of kilonovae can potentially probe increased abundances of Nd. Similarly, at early times when the ejecta is still hot (first day), the U opacity is strong in the $0.2-1$ micron wavelength range and kilonova observations may also be able to constrain these abundances.

\section{INTRODUCTION}

Even before it was known that gamma-ray bursts (GRBs) were of extragalactic origin, astronomers proposed that the merger of either a binary neutron star (NS/NS) or a black hole-neutron star binary (BH/NS) could produce these energetic explosions (Narayan et al. 1992). Under the accretion disk paradigm, these mergers are expected to produce shortduration gamma-ray bursts. By assuming mergers produce the bulk of short-duration GRBs (Popham et al. 1999), population synthesis studies demonstrated that these bursts should occur beyond their star-formation regions, and in some cases, beyond their host galaxies (Fryer et al. 1999; Bloom et al. 1999). The observed spatial distribution matches these predictions (Fong \& Berger 2013), and concurrent gravita-

Corresponding author: W. Even even@lanl.gov tional wave and gamma-ray observations demonstrate that these mergers produce not only gamma-rays (Abbott et al. 2017a,b) but also relativistic jets (Mooley et al. 2018a,b). All of this evidence has gradually produced a near universal acceptance that these compact mergers are behind most short-duration GRBs.

Compact mergers have also been suggested as an r-process site (Lattimer \& Schramm 1974), and increasingly detailed studies support this as a leading source of r-process elements (e.g. Freiburghaus et al. 1999; Korobkin et al. 2012; Bauswein et al. 2013; Radice et al. 2016; Thielemann et al. 2017; Metzger \& Fernández 2014). Models of the r-process-rich merger ejecta coupled with merger rates from the gravitational-wave detection of GW170817 demonstrate that compact mergers could well be the dominant r-process source in the universe (e.g. Abbott et al. 2017c; Côté et al. 2018). However, calculating the ejected mass and its composition is not straightforward. Although the initial ejecta (tidally or dynamically ejected) is very likely to be suffi- 
ciently neutron rich to produce a strong r-process signature, details of the nuclear physics can significantly impact the exact quantities of the isotopes produced (Mumpower et al. 2016, 2018; Zhu et al. 2018; Vassh et al. 2018). After the initial merger, a disk forms that drives further outflows Surman \& McLaughlin (2004); Surman et al. (2006, 2008). If the remnant compact core does not immediately collapse to a black hole, neutron star accretion can also drive outflows. Neutron star accretion has been studied in detail in the context of supernova fallback, and the ejected mass can be as much as $25 \%$ of the accreted material (Fryer et al. 2006; Fryer 2009). The late-time ejecta composition (disk and neutron star ejecta) can be substantially altered by neutrinos, which reset the electron fraction as this material flows outward. With higher electron fractions, the composition can range wildly, producing everything from iron peak elements to transuranic isotopes (Fernández \& Metzger 2013; Just et al. 2015; Bovard et al. 2017; Foucart et al. 2018; Miller et al. 2019). At this time, modeling alone cannot place strong constraints on the precise elemental distributions of merger ejecta.

The optical and infrared observations of GW170817 provided a first glimpse at merger ejecta (Evans et al. 2017; Tanaka et al. 2017; Perego et al. 2017; Smartt et al. 2017; Kasen et al. 2017; Troja et al. 2017; Arcavi et al. 2017; Nicholl et al. 2017; Chornock et al. 2017; Cowperthwaite et al. 2017; Kasliwal et al. 2017; Abbott et al. 2017c). The high optical luminosity in the first day suggested a large amount of low-neutron-fraction (high- $Y_{e}$ ) ejecta with a composition closer to iron peak elements. The late-time (roughly 1 week) peak in the infrared suggested a neutron-rich component (presumably from the dynamical ejecta) that includes a broad lanthanide distribution with high opacities in the optical and near-IR bands. On the surface, it appears that both red and blue ejecta components as outlined by Metzger \& Fernández (2014) are present in GW170817. In principle, features in the optical and infrared observations can be used to probe the ejecta composition, but different studies have predicted very different masses of low- and high-neutronfraction ejecta (for a review, see Côté et al. 2018). One difficulty in inferring the ejecta mass from the observed emission has been differences in the opacities used by the community. For example, some groups use simple, constant opacities while others use opacities derived from detailed atomic physics calculations. Even those calculations that employ detailed opacities typically resort to using a few well-studied elements as surrogates to represent an entire suite of lanthanide opacities.

Without a detailed understanding of the opacities and their effects on the emission, we cannot definitively infer the ejecta composition. In this paper, we conduct the first study of the emission from kilonova using a complete set of lanthanide opacities (Fontes et al. 2019). We use two basic heavyelement profiles based on the solar r-process and simulated merger yields, testing both the case where these heavy elements comprise the bulk of the composition (mimicking dynamical ejecta) and a small fraction of the composition (closer to late-time ejecta). We vary the ejecta composition to search for observational features in the kilonova spectra and light curves that might help observers discriminate between compositions. Section 2 describes our calculations of the ejecta characteristics and details our simulation tools. Section 3 describes the light curves and spectra from our simulation grid, focusing on the physics behind the results that are most sensitive to the composition. Our conclusions summarize the primary results and provide a link to the spectral and light-curve database produced in this study.

\section{CALCULATIONS}

For this sensitivity study, we use a simple 1-dimensional ejecta profile with a homologous outflow. We consider two abundance patterns: one based on solar r-process residuals, and one computed from network calculations of NS merger dynamical ejecta. In this section, we describe this initial profile and the transport model and its assumptions.

\subsection{Initial Conditions}

The late-time ejecta is either driven by disk outflows (neutrino- and viscosity-driven) or neutron star accretion. A number of simulations have been developed to model these outflows (e.g. Fernández \& Metzger 2013; Just et al. 2015; Bovard et al. 2017; Foucart et al. 2018; Miller et al. 2019), each with simplifying assumptions. At this time, no model produces the ejecta properties perfectly (e.g. none develop the highly-relativistic jets observed in GW170817), but models of the bulk of the mass ejected at late times suggest slower velocities than the dynamical ejecta.

The evolution of this homologous outflow is described by the following equations:

$$
\begin{gathered}
v(r, t)=\frac{r}{t} \\
\rho(r, t)=\rho_{0}\left(\frac{t}{t_{0}}\right)^{-3}\left(1-\frac{r^{2}}{\left(v_{\max } t\right)^{2}}\right)^{3}, \\
T(r, t)=T_{0}\left(\frac{t}{t_{0}}\right)^{-1}\left(1-\frac{r^{2}}{\left(v_{\max } t\right)^{2}}\right),
\end{gathered}
$$

where $t_{0}=10,000 \mathrm{~s}$ and $v_{\max }=0.4 c$, with $c$ the speed of light. For an ejecta mass of $M_{\mathrm{ej}}=0.01 \mathrm{M}_{\odot}$, the initial peak density is $\rho_{0}=2.24 \times 10^{-13} \mathrm{~g} / \mathrm{cm}^{3}$. The initial peak temperature $T_{0}$ is set by the residual thermal energy from nucleosynthesis and the energy deposited from the decay of radioactive elements. Following Wollaeger et al. (2018), we set $T_{0}=5.7 \times 10^{4} \mathrm{~K}$.

\subsection{Composition}




\begin{tabular}{l|c|r|r}
\hline \hline element & $\begin{array}{c}\text { atomic } \\
\text { number }\end{array}$ & $\begin{array}{r}\text { dynamcial ejecta }\left(\mathrm{Y}_{e}=0.05\right) \\
\text { mass fraction }\end{array}$ & $\begin{array}{r}\text { solar r-process residuals } \\
\text { mass fraction }\end{array}$ \\
\hline $\mathrm{Fe}$ & 26 & $5.32 \mathrm{e}-6$ & $3.90 \mathrm{e}-2$ \\
$\mathrm{Se}$ & 34 & $1.01 \mathrm{e}-1$ & $4.66 \mathrm{e}-1$ \\
$\mathrm{Br}$ & 35 & $2.32 \mathrm{e}-6$ & $2.65 \mathrm{e}-1$ \\
$\mathrm{Zr}$ & 40 & $3.72 \mathrm{e}-1$ & $3.81 \mathrm{e}-2$ \\
$\mathrm{Pd}$ & 46 & $1.39 \mathrm{e}-4$ & $4.37 \mathrm{e}-2$ \\
$\mathrm{Te}$ & 52 & $3.85 \mathrm{e}-1$ & $1.16 \mathrm{e}-1$ \\
$\mathrm{La}$ & 57 & $5.11 \mathrm{e}-4$ & $1.57 \mathrm{e}-3$ \\
$\mathrm{Ce}$ & 58 & $8.66 \mathrm{e}-4$ & $2.30 \mathrm{e}-3$ \\
$\mathrm{Pr}$ & 59 & $8.59 \mathrm{e}-5$ & $1.12 \mathrm{e}-3$ \\
$\mathrm{Nd}$ & 60 & $1.50 \mathrm{e}-3$ & $3.86 \mathrm{e}-3$ \\
$\mathrm{Pm}$ & 61 & $5.42 \mathrm{e}-4$ & $9.99 \mathrm{e}-4$ \\
$\mathrm{Sm}$ & 62 & $2.03 \mathrm{e}-3$ & $1.98 \mathrm{e}-3$ \\
$\mathrm{Eu}$ & 63 & $1.55 \mathrm{e}-3$ & $1.04 \mathrm{e}-3$ \\
$\mathrm{Gd}$ & 64 & $5.13 \mathrm{e}-3$ & $3.26 \mathrm{e}-3$ \\
$\mathrm{~Tb}$ & 65 & $3.27 \mathrm{e}-3$ & $6.88 \mathrm{e}-4$ \\
$\mathrm{Dy}$ & 66 & $1.40 \mathrm{e}-2$ & $4.26 \mathrm{e}-3$ \\
$\mathrm{Ho}$ & 67 & $3.64 \mathrm{e}-3$ & $9.96 \mathrm{e}-4$ \\
$\mathrm{Er}$ & 68 & $1.11 \mathrm{e}-2$ & $2.62 \mathrm{e}-3$ \\
$\mathrm{Tm}$ & 69 & $2.34 \mathrm{e}-3$ & $4.14 \mathrm{e}-4$ \\
$\mathrm{Yb}$ & 70 & $6.44 \mathrm{e}-3$ & $3.39 \mathrm{e}-3$ \\
$\mathrm{U}$ & 92 & $8.83 \mathrm{e}-2$ & $2.99 \mathrm{e}-3$ \\
\hline
\end{tabular}

Table 1. Effective mass fractions of representative elements used to compute opacities. The third column is the main r-process abundance pattern expected to be produced in dynamical ejecta. The fourth column is the rescaled (by a factor of $3 \times 10^{7}$ ) solar r-process residuals, amended with $\mathrm{Pm}$ given the mass fraction $X_{\mathrm{Pm}}=10^{-3}$. This data is displayed visually in Figure 1.

In these models, we study two different ejecta compositions. The first is based on the solar r-process pattern in the sun, scaling the mass fraction up by $3 \times 10^{7}$ to produce an r-process dominated abundance pattern. Because Pm has no stable isotopes, the solar system has only trace amounts of this element. However, it will be present in the merger, so we also raise the Pm abundance in this model. We term this abundance pattern our "solar r-process" ejecta. Our other composition model consists of the yields from a dynamical ejecta reaction network calculation. These compositions are shown in Figure 1.

Starting from these base compositions, we vary the abundance of individual elements (focusing on lanthanides) to determine the dependence of the light curves and spectra on these species; the results are summarized in Tables 2 and 3. We also vary the total heavy r-process yields to generate models that are closer to what we expect from the late-time ejecta; table 4 summarizes this study.

In calculating our opacities, we employ the full suite of lanthanide opacities from Fontes et al. (2019), but we do not have detailed opacities for the majority of elements lighter and heavier than the lanthanides. For the heavier elements (e.g. actinides), we use uranium as a surrogate. For elements ranging from the iron peak to the lanthanides, we use Fe, Se, $\mathrm{Zr}, \mathrm{Pd}$, and Te as surrogates. For the purpose of computing opacity, the effective abundance of a surrogate element is the sum of the abundances of the elements it represents; these effective abundances are shown in Figure 1 and Table 1.

\subsection{Light-Curve Calculations}

For radiative transfer, we use SuperNu (Wollaeger et al. 2013; Wollaeger \& van Rossum 2014) with tabular opacities from the LANL suite of relativistic atomic physics codes (Fontes et al. 2015). SuperNu is a multidimensional, multi-group Monte Carlo transport method. It has already been used extensively on kilonova light curves in 1- and 2-dimensions to match both the emission from GW170817 (Troja et al. 2017; Tanvir et al. 2017) and in an initial suite of sensitivity studies (Kasliwal et al. 2017; Wollaeger et al. 2018) using a reduced set of opacities from Fontes et al. (2017). For the calculations in this paper, we limit our study to 1-dimensional models with 1024 energy groups. In calculating colors, we use color band filters for the g, r, i z, y, J, H and K bands (Figure 2).

In this project, we used the preferred set of binned opacities from Fontes et al. (2019). Figure 3 shows the structure of the opacities binned at the resolution of our calculations. It shows the opacities of our two base abundances at a temperature of $0.09 \mathrm{eV}$ and a density of $10^{-16} \mathrm{~g} \mathrm{~cm}^{-3}$. The huge number of lines within the wavelength range plotted here (primarily due to the Lanthanides), makes it very difficult to pick out differences. We have binned this forest of lines to the energy resolution in our simulations and, even with this coarse binning, it is difficult to distinguish individual elements via specific lines. In this paper, we examine the dependence of the spectra and light curves on individual lanthanides in order to develop a means to probe the abundances in observed kilonovae.

\section{SPECTRA AND LIGHT CURVES}

Throughout this work, magnitudes and fluxes are computed for an event at a standard distance of $10 \mathrm{pc}$, so magnitudes are absolute magnitudes.

\subsection{Role of Lanthanides}

Before we study the roles of individual lanthanides, we first discuss the impact of lanthanides as a group on kilonova emission spectra and observable light curves. Figure 4 shows the spectra at different times for six different lanthanide abundance fractions; we obtained these abundances from renormalized solar r-process residuals with the lanthanides rescaled by powers of ten, giving lanthanide fractions from $3 \times 10^{-5}$ to 0.75 . Figure 5 shows color band light curves resulting from these spectra. As has been shown by several groups (e.g. Kasen et al. 2017), increased lanthanide fractions reduce the peak in the ultraviolet and optical bands while increasing the emission in the infrared. The $\mathrm{g}$ band peaks strongly at early times if the lanthanide fraction is below $3 \times 10^{-4}$, but higher lanthanide fractions obscure this 

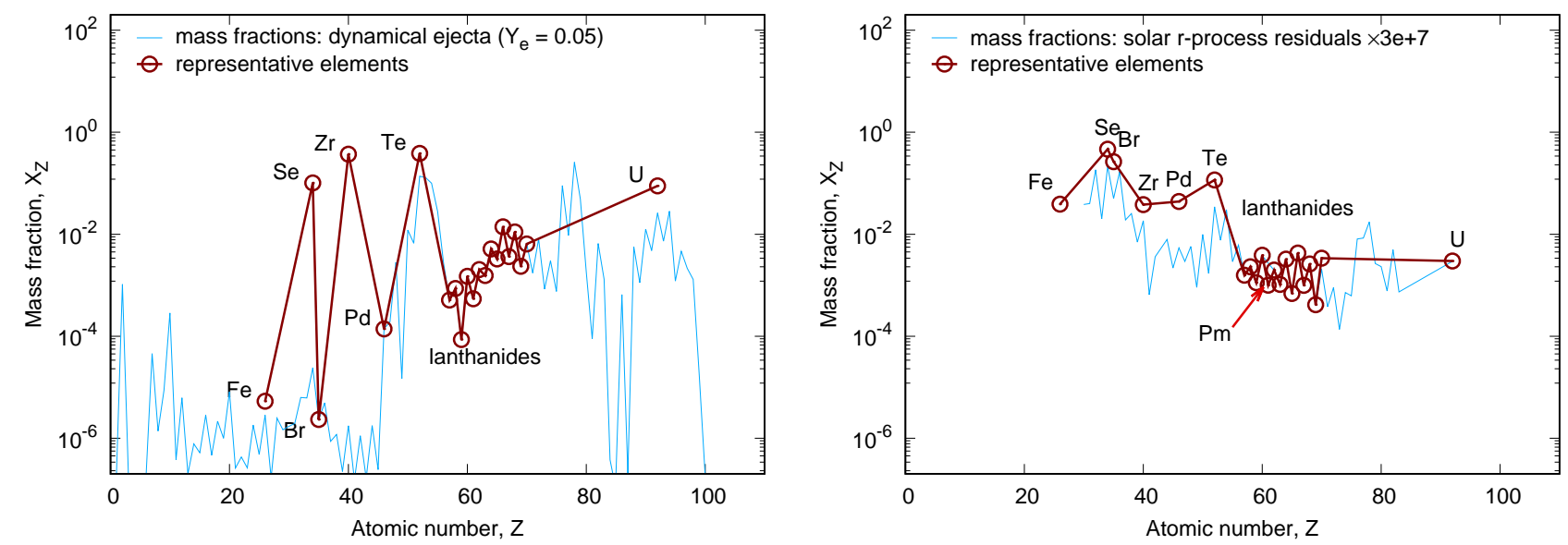

Figure 1. Abundance in mass fraction versus atomic number. Left panel: the main r-process abundance pattern, as expected to be produced in dynamical ejecta; Right panel: rescaled solar r-process residuals, with added Pm with the mass fraction of $X_{\mathrm{Pm}}=10^{-3}$.

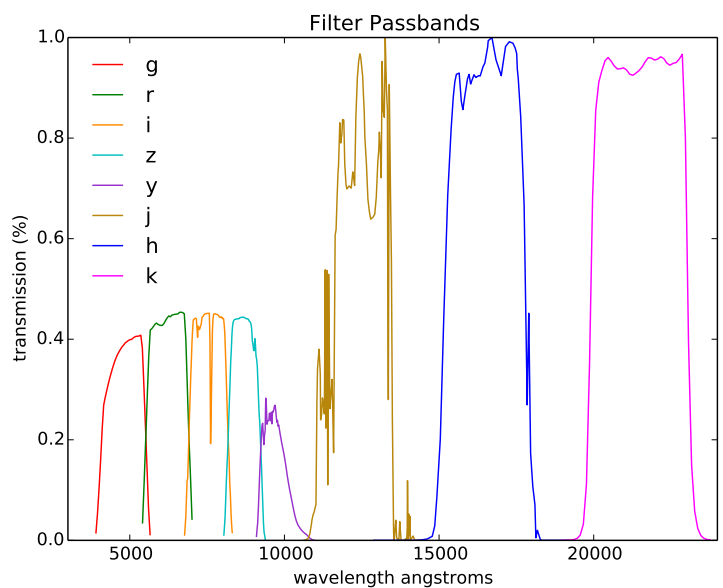

Figure 2. Frequency pass bands used in the analysis of this paper. The optical grizy and nIR $J H K$ passbands are from the LSST and 2MASS filters, respectively.

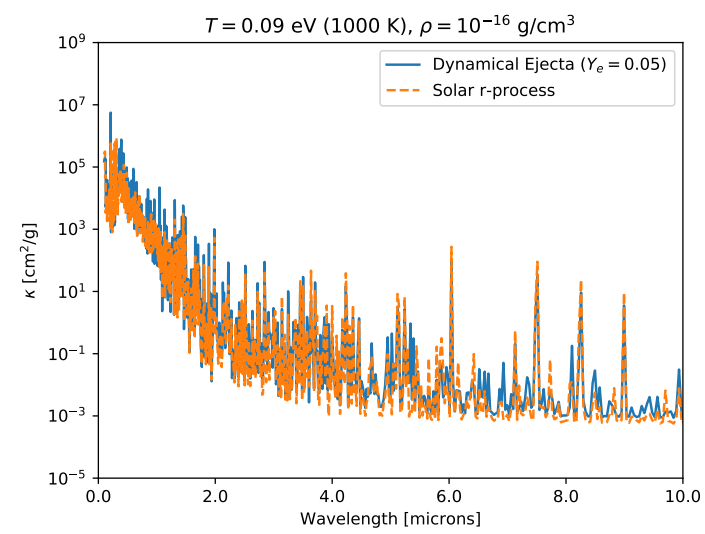

Figure 3. Opacities for our two base abundances: solar and simulation dynamical ejecta at peak. If the lanthanide fraction exceeds $3 \times 10^{-3}$, the peak emission is delayed, reddened, and dimmed. The peak magnitude for high lanthanide fractions occurs in the $\mathrm{K}$ and redder bands, and it is two magnitudes dimmer than the g-band peak for low lanthanide fractions. The infrared peak after $5 \mathrm{~d}$ in GW170817 has been used to argue that lanthanides—and hence heavy r-process elements-were present in its ejecta.

\subsection{Solar r-Process Ejecta}

The focus of this paper is to study the roles of individual lanthanide species in the kilonova spectra. We varied individual lanthanide abundances in our r-process ejecta to search for features specific to each element. Due to the forest of lines in each lanthanide, we do not expect strong line features, but it is possible that the spectra can be affected if one species is over- or under-produced in the ejecta. Such spectral effects, in addition to supplying information about relative abundances, may provide constraints on nuclear physics properties in this nuclear mass region (Mumpower et al. 2016; Mumpower et al. 2017; Orford et al. 2018; Vilén et al. 2018).

Figure 6 shows the effect on kilonova light curves in the $\mathrm{g}$, $\mathrm{r}, \mathrm{z}, \mathrm{J}$, and $\mathrm{K}$ bands for each lanthanide species. We vary the mass fraction of each element up and down by one order of magnitude from the standard value (which is listed at the top of each plot). We include uranium to test the importance of actinides. For the most part, kilonova light curves do not depend significantly on the abundance of individual elements, even as we raise and lower their mass fractions by an order of magnitude. $\mathrm{Nd}$ is a notable exception.

To better understand this dependence, we study the effects of the $\mathrm{Nd}$ abundance in more detail. Figure 7 shows the spectra from our neutron rich simulations when we vary $\mathrm{Nd}$ and $\mathrm{Sm}$ up and down by an order of magnitude while keeping the total lanthanide fraction constant. Most lanthanides behave like Sm in that the spectra between $1-10 \mu \mathrm{m}$ do not de- 


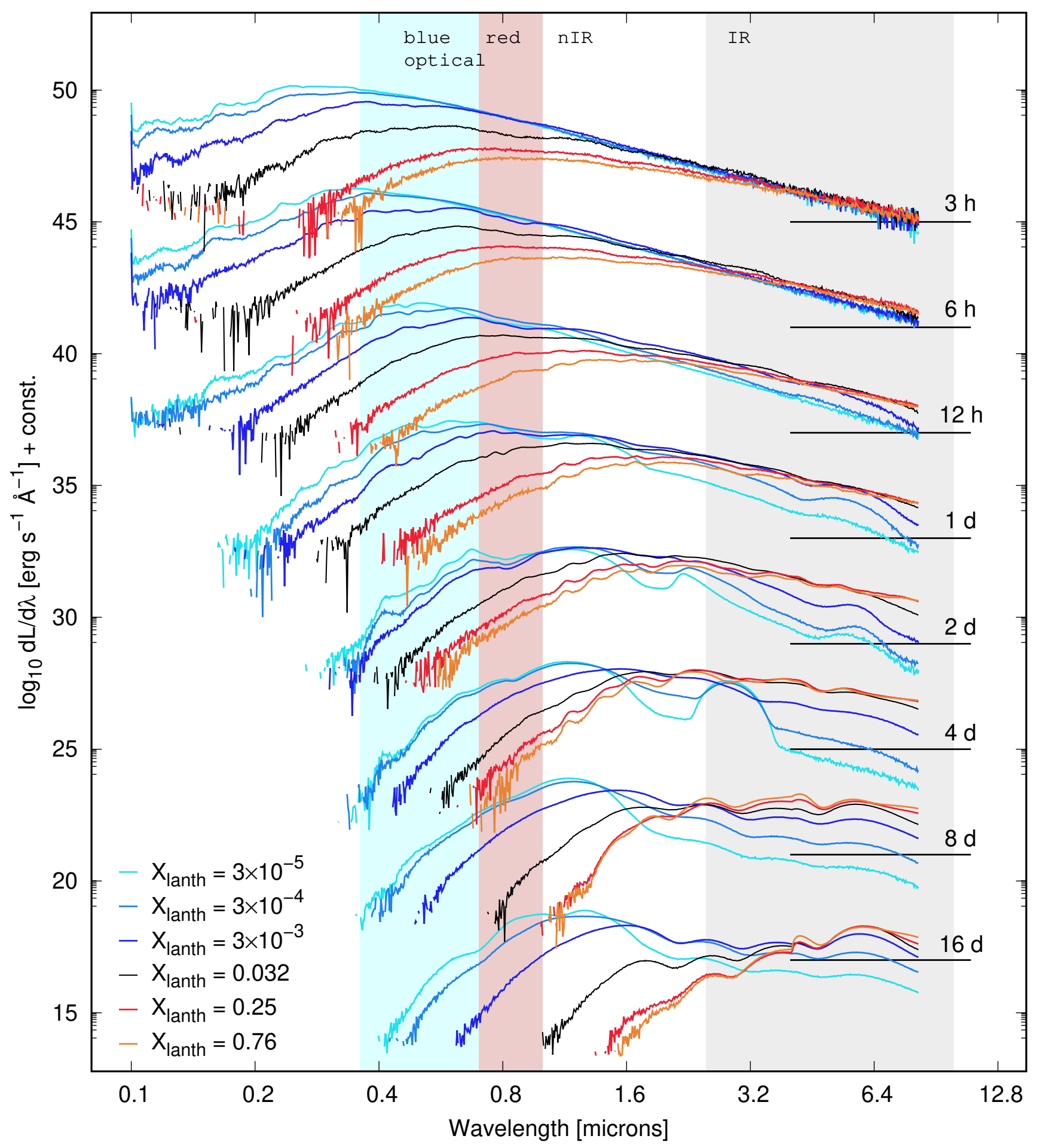

Figure 4. Sensitivity of kilonova spectra to the mass fraction of lanthanides and uranium $(Z \geq 57)$. The baseline model here represents the solar r-process residuals and contains total mass fraction $X_{\text {lanth }}=0.032$ of lanthanides. The constant shifting the y axis is set to zero for the spectra at $1 \mathrm{~d}$. The black horizontal lines correspond to the same flux level for each model (the difference between each line denotes the constant shift). 

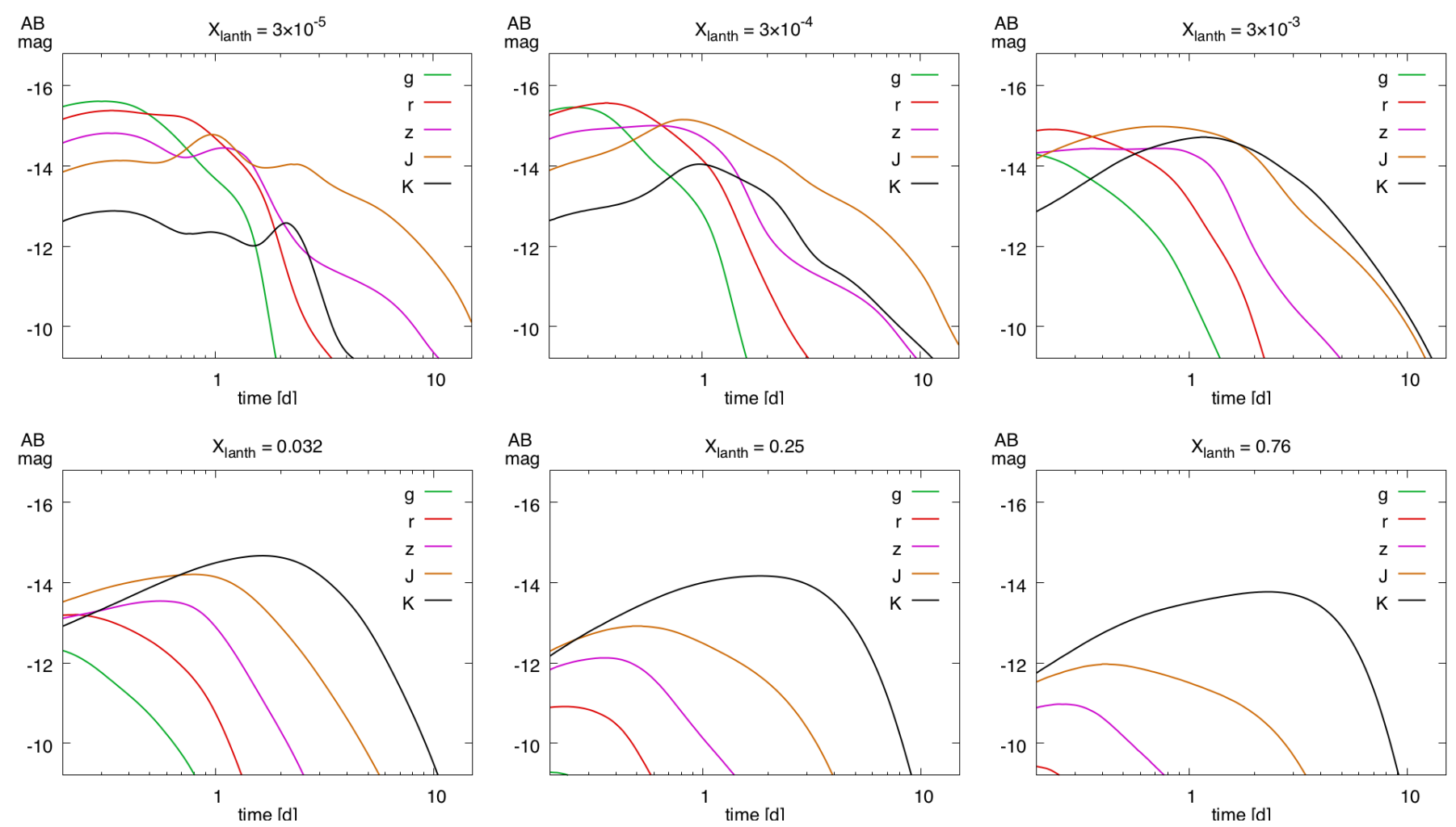

Figure 5. Sensitivity of the broadband light curves to the mass fraction of lanthanides, relative to the solar r-process residuals, in the order of raising lanthanide fraction. 

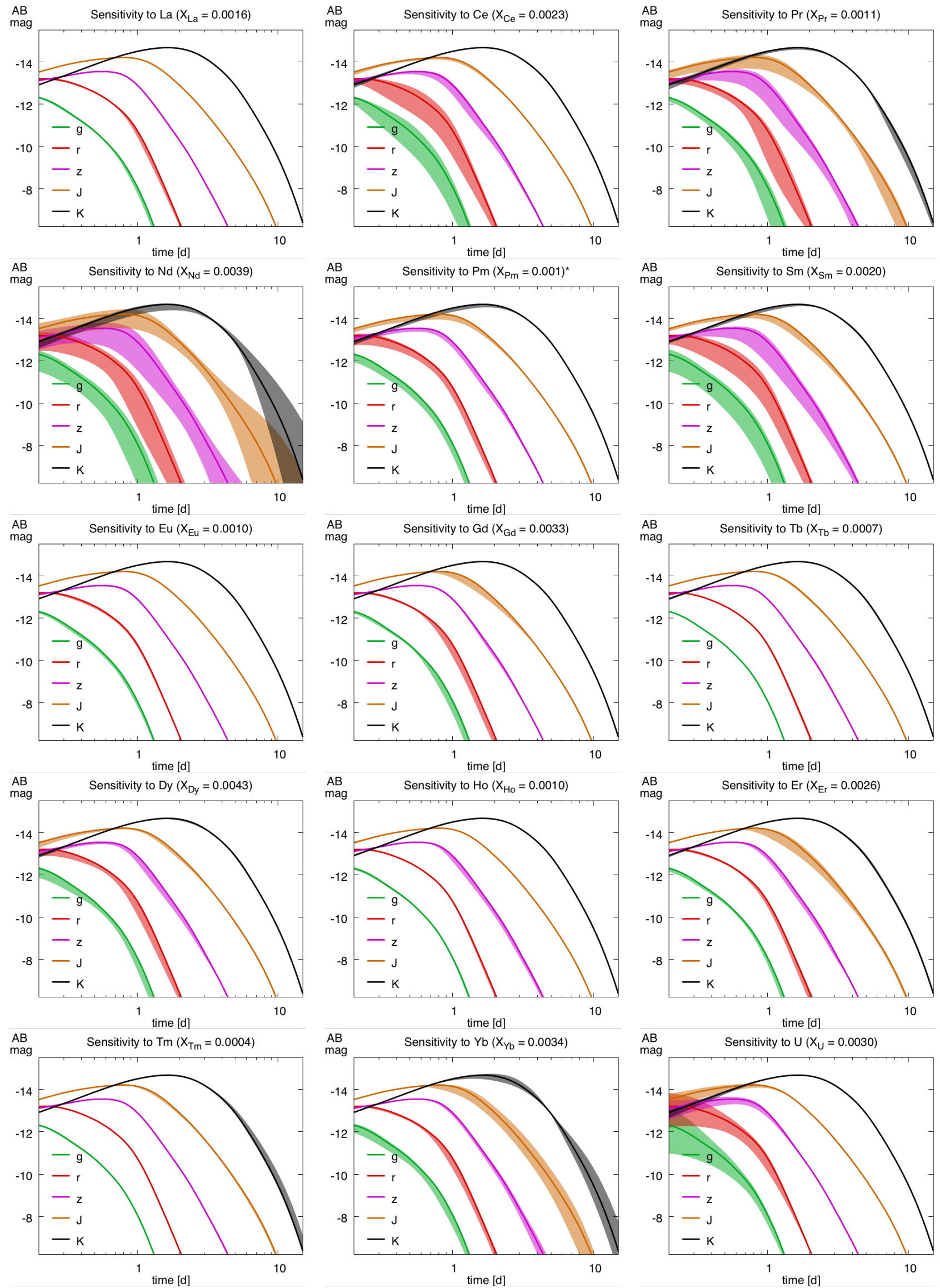

Figure 6. Impact of presence of individual lanthanides on the broadband magnitudes of a kilonova with solar abundances. The value in brackets indicates the mass fraction of a specific element. The range corresponds to the light curve sensitivity to increasing or decreasing the mass fraction by a factor of 10 . 
pend on an individual element's abundance; the thick forest of each species's lines contributes nearly equally across this band. The exception to this basic trend is $\mathrm{Nd}$, which pushes the late-time peak in the emission from $\sim 1 \mu \mathrm{m}$ to $>5 \mu \mathrm{m}$.

The leverage of $\mathrm{Nd}$ on the spectra arises from its high opacity in the near infrared. Figure 8 shows the opacities of $\mathrm{Nd}$ and $\mathrm{Sm}$ between 1 and $10 \mu \mathrm{m}$. Between 1.7 and $4 \mu \mathrm{m}$, the $\mathrm{Nd}$ opacity is nearly an order of magnitude greater. This outsize opacity is a consequence of the energy level diagram for electrically neutral $\mathrm{Nd}$, which is the dominant ionization stage under the relevant conditions. The partially filled $4 f^{4}$ electron ground configuration and the corresponding excited states result in atomic transitions that produce absorption lines in the 1.7 to $4.5 \mu \mathrm{m}$ range; these lines are strong compared to other lanthanides, as illustrated in Figures 6 and 8 of Fontes et al. (2019). In fact, those figures display significantly fewer absorption features from elements in the middle of the lanthanide series, such as Eu and Gd, despite the more complex energy level diagrams associated with their halffilled $4 f^{7}$ ground state configurations. The distinct absorption features of $\mathrm{Nd}$-coupled with its relatively high abundance ( $\mathrm{Nd}$ is an even element)_cause it to dominate the opacity in this region, and adjusting its abundance up/down results in a commensurate increase/decrease in the total opacity. In contrast, increasing the fraction of Sm (an odd element) by a factor of 10 would not even double the opacity. We note that the high $\mathrm{Nd}$ opacity may also explain the redward shift in the calculations by Fontes et al. (2017). That work used $\mathrm{Nd}$ as a surrogate for many of the lanthanides, effectively increasing the opacity in this wavelength band by a factor of a few beyond what we would expect from a full lanthanide distribution.

In addition to the strong dependence on the Nd mass fraction, the early-time light curves $(<1 \mathrm{~d})$ are heavily influenced by the mass fraction of uranium (seen previously in Figure 6). We examined this effect by simulating the light curves with and without uranium. We found that the presence of uranium dramatically lowers the early-time luminosity and suppresses the spectra in the optical and ultraviolet wavelengths. Figure 9 displays these findings.

In order to illuminate these effects, we plot the ejecta opacities with and without uranium at temperatures and densities relevant for early times (Figure 10). Above a temperature of $T \sim 1.0 \mathrm{eV}$, the absorption lines in uranium dominate the opacity from $0.2-1 \mu \mathrm{m}$, obscuring emission in this wavelength range. Similar to $\mathrm{Nd}$, this increase in opacity is related to the energy level diagram of the uranium ion stage that is dominant in the conditions of interest. Note that $U$ is a homologue of $\mathrm{Nd}$, which means they reside in the same column of the periodic table, have similar electron structure, and hence are expected to display similar-though not identical-patterns in their absorption features. Because $U$ is a $\mathrm{Nd}$ analogue, we need to be careful about using it as a surrogate for all actinides, since $\mathrm{Nd}$ behaves differently from other lanthanides.

\subsection{Calculated Abundances}

We repeated our sensitivity study of the color band light curves using abundances from our kilonova dynamical ejecta models. Figure 11 shows the band light curves for these models with each lanthanide (and uranium) abundance varied by a factor of ten up and down from the model abundance. The individual abundance of each lanthanide is different in this composition model from its solar r-process value, and this can alter the effect of varying each by a factor of ten. Nevertheless, the immense influence of the $\mathrm{Nd}$ and $\mathrm{U}$ abundances remains.

\section{SUMMARY}

We presented a complete study of the dependence of the spectra and light curves from a kilonova on the individual lanthanide abundances. For most species, the broad and dense forest of lanthanide lines means that varying its abundance does not affect the light curves or the spectra demonstrably. However, the opacity of $\mathrm{Nd}$ is singularly dominant in the $\sim 1 \mu \mathrm{m}$ to $\sim 5 \mu \mathrm{m}$ range, and variations in its abundance can dramatically alter the color band light curves. The abundance of $\mathrm{Nd}$ also moves the peak wavelength of the infrared emission, causing it to vary from $\sim 1.6 \mu \mathrm{m}$ for low $\mathrm{Nd}$ abundances to $\sim 6 \mu \mathrm{m}$ for high $\mathrm{Nd}$ abundances. If we can sufficiently reduce the uncertainties in the ejecta properties, we can infer the abundance fraction of $\mathrm{Nd}$ by measuring this peak.

The spectra and light-curve data from these calculations is available at the Center for Theoretical Astrophysics website: https://ccsweb.lanl.gov/astro/transient/transients_astro.html.

\section{ACKNOWLEDGMENTS}

This work was supported by the US Department of Energy through the Los Alamos National Laboratory. Los Alamos National Laboratory is operated by Triad National Security, LLC, for the National Nuclear Security Administration of U.S. Department of Energy (Contract No. 89233218CNA000001). Research presented in this article was supported by the Laboratory Directed Research and Development program of Los Alamos National Laboratory under project number 20190021DR. 

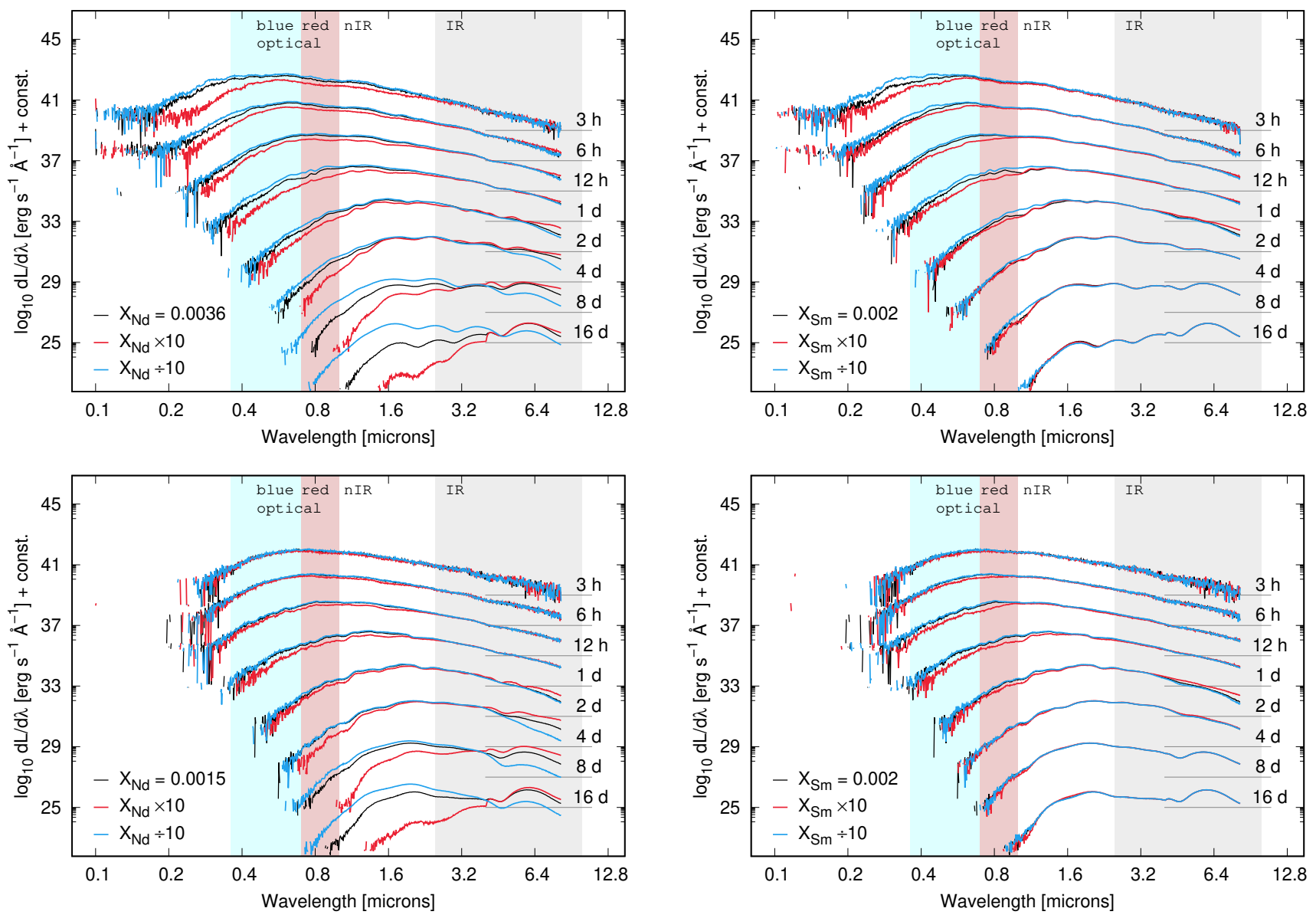

Figure 7. Dependence of kilonova spectra on the mass fractions of Nd (left) and Sm (right) at a range of times. The mass fractions of these individual species vary up and down by an order of magnitude; the total lanthanide fraction is kept constant. The top panels show variation from solar r-process residuals, and the bottom panels show variation from the main r-process (second to third peak) abundance pattern. As with most lanthanides, varying Sm does not dramatically alter the kilonova spectra. However, Nd can dramatically shift the peak of the emission at late times (from $\sim 1 \mu \mathrm{m}$ to $>5 \mu \mathrm{m}$ )

\section{APPENDIX}

\section{A. MODEL TABLES: PEAK MAGNITUDES, EPOCHS AND DURATIONS}

In this section, we tabulate properties of our models. These tables are divided into left and right sections, where each column of each section contains a grizyJHK filter. The left section contains peak magnitudes, and the right section contains [time of peak magnitude] / [duration of peak magnitude]. Following Wollaeger et al. (2018), the duration of the peak is defined as the time it takes for that particular broadband light curve to drop in brightness by one magnitude.

\section{REFERENCES}

Abbott, B. P., Abbott, R., Abbott, T. D., et al. 2017a, ApJL, 848,

L12

-. 2017b, ApJL, 848, L13

—. 2017c, ApJL, 850, L39

Arcavi, I., Hosseinzadeh, G., Howell, D. A., et al. 2017, Nature, 551,64
Bauswein, A., Goriely, S., \& Janka, H.-T. 2013, ApJ, 773, 78

Bloom, J. S., Sigurdsson, S., \& Pols, O. R. 1999, MNRAS, 305, 763

Bovard, L., Martin, D., Guercilena, F., et al. 2017, PhRvD, 96, 124005

Chornock, R., Berger, E., Kasen, D., et al. 2017, ApJL, 848, L19 

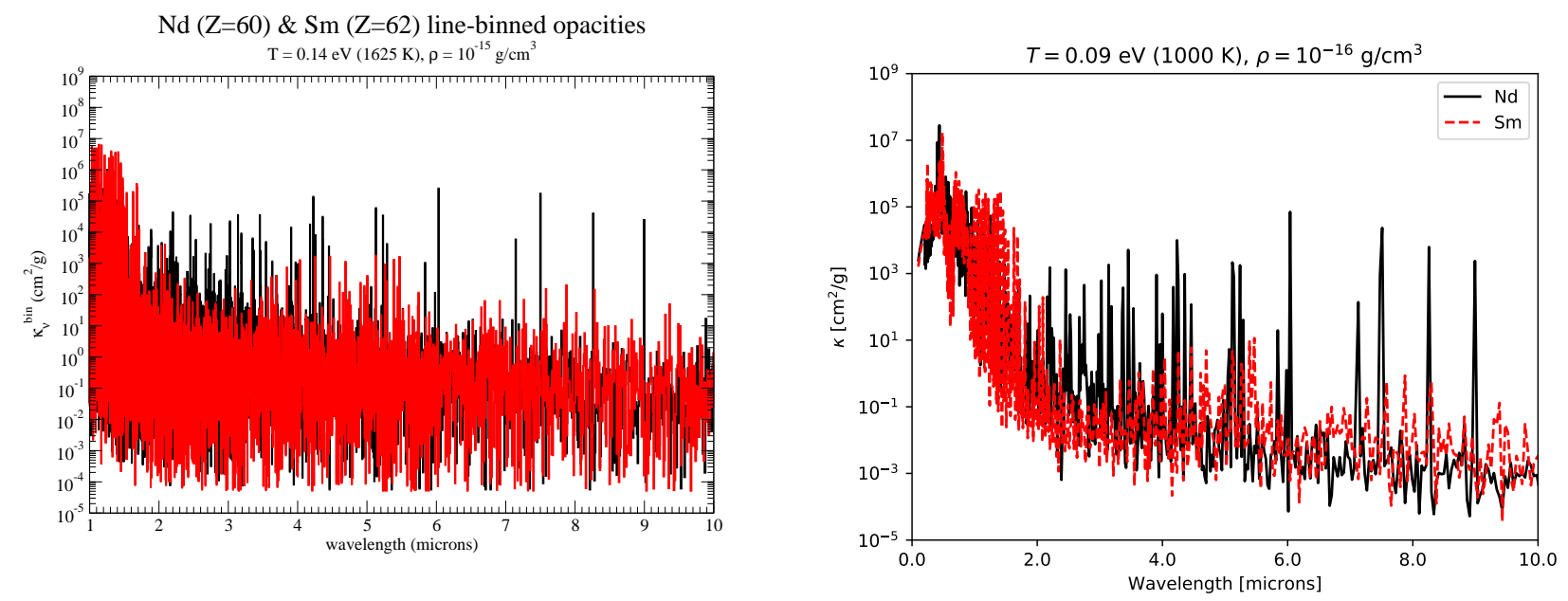

Figure 8. Opacity of $\mathrm{Nd}$ (black) versus $\mathrm{Sm}$ (red) in the wavelength range from $1-10 \mu \mathrm{m}$. Between $1.7-4 \mu \mathrm{m}$, the Nd opacity is has roughly an order of magnitude greater opacity. The left panel shows the full opacity from Fontes et al. (2019), and the right panel shows this opacity once it is binned at the energy resolution of the simulations.
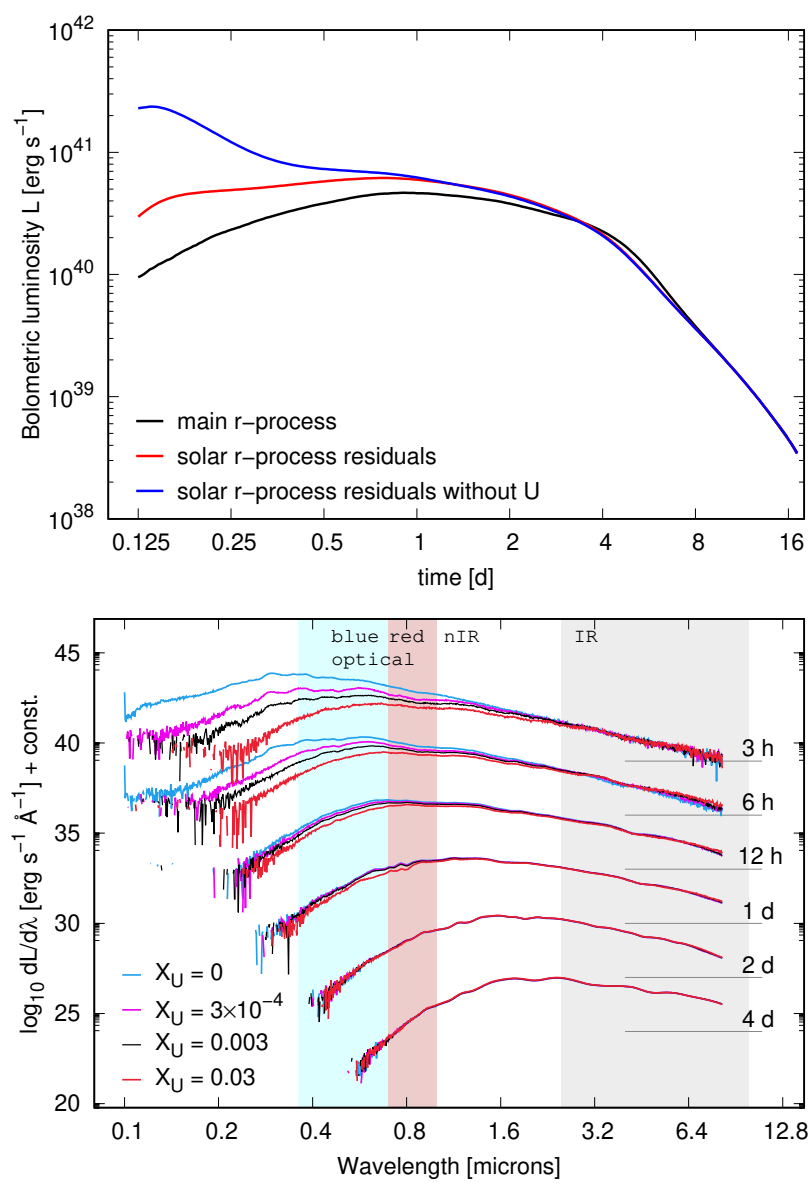

Figure 9. Impact of uranium on bolometric luminosity and spectra. Black: synthetic main r-process composition. Red: solar r-process residuals. Blue: solar r-process composition with the uranium removed.
Côté, B., Fryer, C. L., Belczynski, K., et al. 2018, ApJ, 855, 99

Cowperthwaite, P. S., Berger, E., Villar, V. A., et al. 2017, ApJL, 848, L17

Evans, P. A., Cenko, S. B., Kennea, J. A., et al. 2017, Science, 358, 1565

Fernández, R., \& Metzger, B. D. 2013, MNRAS, 435, 502

Fong, W., \& Berger, E. 2013, ApJ, 776, 18

Fontes, C. J., Fryer, C. L., Hungerford, A. L., Wollaeger, R. T., \& Korobkin, O. 2019, arXiv e-prints, arXiv:1904.08781

Fontes, C. J., Fryer, C. L., Hungerford, A. L., et al. 2017, arXiv e-prints, arXiv: 1702.02990

Fontes, C. J., Zhang, H. L., Abdallah, J., et al. 2015, Journal of Physics B: Atomic, Molecular and Optical Physics, 48, 144014

Foucart, F., Duez, M. D., Kidder, L. E., et al. 2018, PhRvD, 98, 063007

Freiburghaus, C., Rosswog, S., \& Thielemann, F.-K. 1999, ApJL, 525, L121. http://adsabs.harvard.edu/abs/1999ApJ...525L.121F

Fryer, C. L. 2009, ApJ, 699, 409

Fryer, C. L., Woosley, S. E., \& Hartmann, D. H. 1999, ApJ, 526, 152

Fryer, C. L., Young, P. A., \& Hungerford, A. L. 2006, ApJ, 650, 1028

Just, O., Bauswein, A., Ardevol Pulpillo, R., Goriely, S., \& Janka, H.-T. 2015, MNRAS, 448, 541

Kasen, D., Metzger, B., Barnes, J., Quataert, E., \& Ramirez-Ruiz, E. 2017, Nature, 551, 80

Kasliwal, M. M., Korobkin, O., Lau, R. M., Wollaeger, R., \& Fryer, C. L. 2017, ApJL, 843, L34

Korobkin, O., Rosswog, S., Arcones, A., \& Winteler, C. 2012, MNRAS, 426, 1940

Lattimer, J. M., \& Schramm, D. N. 1974, ApJL, 192, L145. http://adsabs.harvard.edu/abs/1974ApJ...192L.145L 
Table 2. Properties of light curves for $2-3$ peak dynamical ejecta abundance. The naming convention is "SALanth_xx_[xo]10", which implies element "xx" is multiplied ("x10") or divided ("o10") by 10 in the baseline 2-3 peak composition.

\begin{tabular}{|c|c|c|c|c|c|c|c|c|c|c|c|c|c|c|c|c|}
\hline \multirow[b]{2}{*}{ Model } & \multicolumn{8}{|c|}{ Peak magnitude, $m$} & \multicolumn{8}{|c|}{ Peak epoch $t_{p}[\mathrm{~d}] /$ duration $\Delta t_{1 \mathrm{mag}}[\mathrm{d}]$} \\
\hline & $\mathrm{g}$ & $\mathrm{r}$ & $\mathrm{i}$ & $\mathrm{z}$ & $\mathrm{y}$ & $\mathrm{J}$ & $\mathrm{H}$ & $\mathrm{K}$ & $\mathrm{g}$ & $\mathrm{r}$ & $\mathrm{i}$ & $\mathrm{z}$ & $\mathrm{y}$ & $\mathrm{J}$ & $\mathrm{H}$ & $\mathrm{K}$ \\
\hline SALanth_br_o10 & -10.2 & -11.8 & -12.8 & -13.2 & -13.5 & -14.1 & -14.4 & -14.5 & $0.23 / 0.60$ & $0.31 / 0.70$ & $0.42 / 0.78$ & $0.57 / 1.00$ & $0.63 / 1.10$ & $0.82 / 1.60$ & $1.18 / 2.82$ & $1.82 / 4.66$ \\
\hline SALanth_br_x10 & -10.2 & -11.8 & -12.8 & -13.2 & -13.5 & -14.2 & -14.4 & -14.5 & $0.23 / 0.59$ & $0.28 / 0.69$ & $0.43 / 0.79$ & $0.56 / 1.00$ & $0.64 / 1.10$ & $0.78 / 1.59$ & $1.17 / 2.82$ & $1.75 / 4.65$ \\
\hline SALanth_ce_o10 & -10.2 & -11.8 & -12.8 & -13.3 & -13.6 & -14.2 & -14.5 & -14.5 & $0.24 / 0.61$ & $0.31 / 0.71$ & $0.43 / 0.79$ & $0.55 / 1.01$ & $0.64 / 1.10$ & $0.80 / 1.58$ & $1.15 / 2.80$ & $1.76 / 4.65$ \\
\hline SALanth_ce_x10 & -9.8 & -11.4 & -12.6 & -13.1 & -13.4 & -14.1 & -14.4 & -14.5 & $0.22 / 0.58$ & $0.27 / 0.71$ & $0.38 / 0.77$ & $0.52 / 0.98$ & $0.60 / 1.08$ & $0.78 / 1.61$ & $1.14 / 2.86$ & $1.80 / 4.70$ \\
\hline SALanth_dy_o10 & -10.2 & -11.8 & -12.8 & -13.2 & -13.5 & -14.2 & -14.5 & -14.5 & $0.22 / 0.60$ & $0.31 / 0.70$ & $0.44 / 0.78$ & $0.56 / 1.01$ & $0.63 / 1.10$ & $0.79 / 1.61$ & $1.18 / 2.84$ & $1.80 / 4.65$ \\
\hline SALanth_dy_x10 & -10.0 & -11.7 & -12.7 & -13.1 & -13.5 & -14.1 & -14.3 & -14.4 & $0.26 / 0.58$ & $0.30 / 0.66$ & $0.41 / 0.71$ & $0.52 / 0.88$ & $0.60 / 1.01$ & $0.72 / 1.43$ & $0.94 / 2.83$ & $1.36 / 4.91$ \\
\hline SALanth_er_o10 & -10.2 & -11.8 & -12.8 & -13.2 & -13.6 & -14.2 & -14.4 & -14.5 & $0.25 / 0.60$ & $0.31 / 0.70$ & $0.44 / 0.79$ & $0.53 / 1.01$ & $0.68 / 1.21$ & $0.88 / 1.90$ & $1.09 / 2.83$ & $1.78 / 4.64$ \\
\hline SALanth_er_x10 & -10.1 & -11.8 & -12.8 & -13.2 & -13.3 & -13.9 & -14.5 & -14.5 & $0.23 / 0.61$ & $0.30 / 0.71$ & $0.44 / 0.77$ & $0.53 / 0.93$ & $0.46 / 0.99$ & $0.60 / 1.33$ & $1.12 / 2.70$ & $1.69 / 4.69$ \\
\hline SALanth_eu_o10 & -10.2 & -11.8 & -12.8 & -13.2 & -13.6 & -14.2 & -14.4 & -14.5 & $0.22 / 0.60$ & $0.30 / 0.71$ & $0.42 / 0.78$ & $0.57 / 1.00$ & $0.64 / 1.09$ & $0.80 / 1.59$ & $1.17 / 2.81$ & $1.83 / 4.65$ \\
\hline SALanth_eu_x10 & -10.1 & -11.7 & -12.7 & -13.1 & -13.4 & -14.1 & -14.4 & -14.5 & $0.23 / 0.57$ & $0.31 / 0.69$ & $0.40 / 0.77$ & $0.56 / 0.99$ & $0.63 / 1.13$ & $0.79 / 1.61$ & $1.15 / 2.85$ & $1.81 / 4.69$ \\
\hline SALanth_fe_o10 & -10.1 & -11.8 & -12.8 & -13.2 & -13.5 & -14.2 & -14.4 & -14.5 & $0.21 / 0.61$ & $0.30 / 0.70$ & $0.43 / 0.78$ & $0.54 / 1.00$ & $0.65 / 1.10$ & $0.79 / 1.59$ & $1.19 / 2.82$ & $1.79 / 4.66$ \\
\hline SALanth_fe_x10 & -10.1 & -11.8 & -12.8 & -13.2 & -13.5 & -14.2 & -14.4 & -14.5 & $0.22 / 0.60$ & $0.31 / 0.70$ & $0.42 / 0.78$ & $0.55 / 1.00$ & $0.64 / 1.10$ & $0.82 / 1.60$ & $1.17 / 2.81$ & $1.81 / 4.66$ \\
\hline SALanth_gd_o10 & -10.2 & -11.8 & -12.8 & -13.2 & -13.5 & -14.2 & -14.4 & -14.5 & $0.22 / 0.60$ & $0.32 / 0.70$ & $0.42 / 0.78$ & $0.55 / 1.00$ & $0.65 / 1.10$ & $0.80 / 1.62$ & $1.18 / 2.83$ & $1.84 / 4.66$ \\
\hline SALanth_gd_x10 & -10.1 & -11.8 & -12.8 & -13.2 & -13.5 & -14.1 & -14.5 & -14.5 & $0.23 / 0.57$ & $0.31 / 0.68$ & $0.43 / 0.78$ & $0.59 / 0.99$ & $0.59 / 1.05$ & $0.70 / 1.45$ & $1.15 / 2.76$ & $1.67 / 4.72$ \\
\hline SALanth_ho_o10 & -10.1 & -11.8 & -12.8 & -13.2 & -13.5 & -14.2 & -14.4 & -14.5 & $0.27 / 0.60$ & $0.30 / 0.70$ & $0.44 / 0.78$ & $0.57 / 1.01$ & $0.64 / 1.12$ & $0.77 / 1.60$ & $1.18 / 2.82$ & $1.80 / 4.65$ \\
\hline SALanth_ho_x10 & -10.1 & -11.8 & -12.8 & -13.2 & -13.5 & -14.2 & -14.4 & -14.5 & $0.26 / 0.62$ & $0.31 / 0.72$ & $0.43 / 0.77$ & $0.52 / 0.91$ & $0.59 / 1.01$ & $0.79 / 1.54$ & $1.13 / 2.78$ & $1.67 / 4.71$ \\
\hline SALanth_la_o10 & -10.2 & -11.8 & -12.8 & -13.2 & -13.6 & -14.2 & -14.4 & -14.5 & $0.24 / 0.60$ & $0.31 / 0.70$ & $0.43 / 0.78$ & $0.55 / 1.00$ & $0.64 / 1.09$ & $0.79 / 1.59$ & $1.15 / 2.82$ & $1.82 / 4.65$ \\
\hline SALanth_la_x10 & -10.1 & -11.7 & -12.8 & -13.2 & -13.5 & -14.2 & -14.4 & -14.5 & $0.24 / 0.60$ & $0.31 / 0.70$ & $0.44 / 0.78$ & $0.59 / 1.01$ & $0.65 / 1.10$ & $0.80 / 1.59$ & $1.17 / 2.81$ & $1.80 / 4.66$ \\
\hline SALanth_nd_o10 & -10.2 & -11.8 & -12.9 & -13.4 & -13.7 & -14.3 & -14.6 & -14.5 & $0.26 / 0.63$ & $0.31 / 0.74$ & $0.43 / 0.81$ & $0.56 / 1.03$ & $0.69 / 1.12$ & $0.82 / 1.58$ & $1.18 / 2.58$ & $1.80 / 4.79$ \\
\hline SALanth_nd_x10 & -10.0 & -11.5 & -12.4 & -12.7 & -13.0 & -13.6 & -14.0 & -14.4 & $0.23 / 0.51$ & $0.28 / 0.60$ & $0.38 / 0.69$ & $0.45 / 0.88$ & $0.53 / 1.01$ & $0.69 / 1.70$ & $1.17 / 3.19$ & $1.87 / 4.65$ \\
\hline SALanth_pd_o10 & -10.1 & -11.8 & -12.8 & -13.2 & -13.5 & -14.2 & -14.4 & -14.5 & $0.28 / 0.61$ & $0.31 / 0.71$ & $0.44 / 0.78$ & $0.56 / 1.00$ & $0.65 / 1.10$ & $0.79 / 1.59$ & $1.16 / 2.81$ & $1.83 / 4.66$ \\
\hline SALanth_pd_x10 & -10.2 & -11.8 & -12.8 & -13.2 & -13.5 & -14.2 & -14.4 & -14.5 & $0.24 / 0.60$ & $0.31 / 0.70$ & $0.43 / 0.78$ & $0.54 / 1.00$ & $0.64 / 1.10$ & $0.79 / 1.60$ & $1.15 / 2.82$ & $1.82 / 4.66$ \\
\hline SALanth_pm_o10 & -10.2 & -11.8 & -12.8 & -13.3 & -13.6 & -14.2 & -14.5 & -14.5 & $0.25 / 0.61$ & $0.31 / 0.70$ & $0.42 / 0.78$ & $0.57 / 1.00$ & $0.64 / 1.10$ & $0.80 / 1.59$ & $1.17 / 2.79$ & $1.76 / 4.64$ \\
\hline SALanth_pm_x10 & -10.1 & -11.6 & -12.7 & -13.1 & -13.4 & -14.0 & -14.3 & -14.5 & $0.21 / 0.57$ & $0.32 / 0.67$ & $0.39 / 0.75$ & $0.53 / 0.96$ & $0.61 / 1.05$ & $0.77 / 1.63$ & $1.16 / 2.98$ & $1.91 / 4.78$ \\
\hline SALanth_pr_o10 & -10.2 & -11.8 & -12.8 & -13.3 & -13.6 & -14.2 & -14.5 & -14.5 & $0.23 / 0.61$ & $0.30 / 0.70$ & $0.44 / 0.78$ & $0.56 / 1.00$ & $0.65 / 1.10$ & $0.79 / 1.59$ & $1.16 / 2.80$ & $1.79 / 4.65$ \\
\hline SALanth_pr_x10 & -10.1 & -11.7 & -12.7 & -13.1 & -13.4 & -14.0 & -14.3 & -14.5 & $0.26 / 0.58$ & $0.29 / 0.67$ & $0.41 / 0.76$ & $0.53 / 0.96$ & $0.61 / 1.09$ & $0.77 / 1.60$ & $1.16 / 2.94$ & $1.82 / 4.70$ \\
\hline SALanth_se_o10 & -10.0 & -11.7 & -12.7 & -13.2 & -13.5 & -14.1 & -14.4 & -14.5 & $0.25 / 0.60$ & $0.30 / 0.69$ & $0.41 / 0.77$ & $0.54 / 0.97$ & $0.61 / 1.07$ & $0.77 / 1.57$ & $1.15 / 2.84$ & $1.75 / 4.70$ \\
\hline SALanth_se_x10 & -10.9 & -12.4 & -13.3 & -13.7 & -13.9 & -14.5 & -14.7 & -14.6 & $0.24 / 0.65$ & $0.37 / 0.81$ & $0.52 / 0.90$ & $0.67 / 1.17$ & $0.80 / 1.30$ & $0.94 / 1.76$ & $1.28 / 2.75$ & $1.97 / 4.51$ \\
\hline SALanth_sm_o10 & -10.2 & -11.9 & -13.0 & -13.4 & -13.6 & -14.2 & -14.5 & -14.5 & $0.26 / 0.64$ & $0.30 / 0.72$ & $0.46 / 0.81$ & $0.60 / 1.03$ & $0.68 / 1.13$ & $0.82 / 1.62$ & $1.21 / 2.79$ & $1.81 / 4.61$ \\
\hline SALanth_sm_x10 & -9.7 & -11.3 & -12.1 & -12.7 & -13.2 & -13.9 & -14.3 & -14.4 & $0.19 / 0.49$ & $0.26 / 0.61$ & $0.35 / 0.74$ & $0.42 / 0.89$ & $0.52 / 0.90$ & $0.70 / 1.50$ & $1.09 / 3.03$ & $1.84 / 4.89$ \\
\hline SALanth_tb_o10 & -10.1 & -11.8 & -12.8 & -13.2 & -13.5 & -14.2 & -14.4 & & $0.26 / 0.61$ & $0.30 / 0.70$ & $0.43 / 0.78$ & $0.56 / 1.00$ & $0.63 / 1.10$ & $0.79 / 1.61$ & $1.18 / 2.84$ & $1.82 / 4.65$ \\
\hline SALanth_tb_x10 & -10.1 & -11.8 & -12.8 & -13.2 & -13.5 & & & -14.5 & $0.24 / 0.59$ & $0.31 / 0.69$ & $0.42 / 0.77$ & $0.57 / 0.99$ & $0.64 / 1.07$ & $0.74 / 1.46$ & $1.05 / 2.66$ & $1.68 / 4.73$ \\
\hline SALanth_te_o10 & -9.7 & -11.3 & -12.4 & -12.9 & -13.2 & -13.9 & -14.2 & -14.4 & $0.22 / 0.57$ & $0.28 / 0.65$ & $0.38 / 0.73$ & $0.49 / 0.91$ & $0.54 / 0.99$ & $0.70 / 1.50$ & $1.09 / 2.89$ & $1.63 / 4.85$ \\
\hline SALanth_te_x10 & -11.7 & -13.0 & -13.8 & -14.1 & -14.3 & -14.8 & -14.8 & -14.7 & $0.21 / 0.71$ & $0.43 / 0.99$ & $0.60 / 1.11$ & $0.79 / 1.40$ & $0.96 / 1.59$ & $1.08 / 2.03$ & $1.35 / 2.80$ & $1.17 / 4.18$ \\
\hline SALanth_tm_o10 & -10.1 & -11.8 & -12.8 & -13.2 & -13.5 & -14.2 & -14.5 & -14.5 & $0.27 / 0.61$ & $0.30 / 0.70$ & $0.44 / 0.78$ & $0.56 / 1.00$ & $0.64 / 1.09$ & $0.80 / 1.61$ & $1.20 / 2.96$ & $1.83 / 4.60$ \\
\hline SALanth_tm_x10 & -10.1 & -11.7 & -12.8 & -13.2 & -13.5 & -14.0 & -14.3 & -14.5 & $0.24 / 0.61$ & $0.29 / 0.69$ & $0.42 / 0.78$ & $0.58 / 0.99$ & $0.61 / 1.08$ & $0.71 / 1.57$ & $0.92 / 2.46$ & $1.57 / 4.97$ \\
\hline SALanth_u_o10 & -11.6 & -12.8 & -13.3 & -13.6 & -13.8 & -14.4 & -14.5 & -14.5 & $0.18 / 0.45$ & $0.26 / 0.63$ & $0.41 / 0.85$ & $0.59 / 1.01$ & $0.65 / 1.09$ & $0.76 / 1.46$ & $1.13 / 2.66$ & $1.69 / 4.58$ \\
\hline SALanth_u_x10 & -9.1 & -10.8 & -12.0 & -12.7 & -13.1 & -13.9 & -14.5 & -14.6 & $0.27 / 0.59$ & $0.27 / 0.73$ & $0.36 / 0.83$ & $0.48 / 1.03$ & $0.62 / 1.20$ & $0.87 / 1.97$ & $1.27 / 3.13$ & $2.06 / 4.67$ \\
\hline SALanth_yb_o10 & -10.2 & -11.8 & -12.8 & -13.2 & -13.6 & -14.2 & -14.6 & -14.7 & $0.26 / 0.59$ & $0.32 / 0.70$ & $0.44 / 0.79$ & $0.54 / 1.00$ & $0.64 / 1.11$ & $0.82 / 1.65$ & $1.31 / 3.01$ & $2.14 / 4.56$ \\
\hline SALanth_yb_x10 & -9.9 & -11.7 & -12.8 & -13.3 & -13.5 & -13.9 & -14.2 & -14.1 & $0.23 / 0.60$ & $0.30 / 0.69$ & $0.44 / 0.77$ & $0.58 / 0.97$ & $0.61 / 1.06$ & $0.66 / 1.55$ & $0.95 / 2.85$ & $1.17 / 4.77$ \\
\hline SALanth_zr_o10 & -9.8 & -11.5 & -12.5 & -13.1 & -13.3 & -13.9 & -14.3 & -14.5 & $0.29 / 0.61$ & $0.33 / 0.69$ & $0.43 / 0.80$ & $0.56 / 1.02$ & $0.58 / 1.07$ & $0.72 / 1.52$ & $1.09 / 2.86$ & $1.45 / 4.66$ \\
\hline SALanth_zr_x10 & -11.6 & -12.9 & -13.6 & -13.9 & -14.1 & -14.7 & -14.8 & -14.5 & $0.22 / 0.56$ & $0.35 / 0.75$ & $0.52 / 0.94$ & $0.68 / 1.11$ & $0.78 / 1.37$ & $0.95 / 1.98$ & $1.39 / 2.81$ & $1.03 / 4.60$ \\
\hline
\end{tabular}

Metzger, B. D., \& Fernández, R. 2014, MNRAS, 441, 3444

Miller, J. M., Ryan, B. R., \& Dolence, J. C. 2019, ApJS, 241, 30

Mooley, K. P., Deller, A. T., Gottlieb, O., et al. 2018a, Nature, 561, 355

Mooley, K. P., Frail, D. A., Dobie, D., et al. 2018b, ApJL, 868, L11

Mumpower, M. R., Kawano, T., Sprouse, T. M., et al. 2018, ApJ, 869,14

Mumpower, M. R., McLaughlin, G., Surman, R., \& Steiner, A. W. 2017, Journal of Physics G: Nuclear and Particle Physics, 44, 034003

Mumpower, M. R., Surman, R., McLaughlin, G. C., \& Aprahamian, A. 2016, Progress in Particle and Nuclear Physics, 86,86
Narayan, R., Paczynski, B., \& Piran, T. 1992, ApJL, 395, L83

Nicholl, M., Berger, E., Kasen, D., et al. 2017, ApJL, 848, L18

Orford, R., Vassh, N., Clark, J., et al. 2018, Physical review letters, 120, 262702

Perego, A., Radice, D., \& Bernuzzi, S. 2017, ApJL, 850, L37

Popham, R., Woosley, S. E., \& Fryer, C. 1999, ApJ, 518, 356

Radice, D., Galeazzi, F., Lippuner, J., et al. 2016, MNRAS, 460, 3255

Smartt, S. J., Chen, T.-W., Jerkstrand, A., et al. 2017, Nature, 551, 75

Surman, R., \& McLaughlin, G. C. 2004, ApJ, 603, 611

Surman, R., McLaughlin, G. C., \& Hix, W. R. 2006, ApJ, 643, 1057 
Table 3. Properties of light curves for solar r-process abundance, plus Pm and U. The naming convention is "SolarUPm_xx_[xo]10", which implies element "xx" is multiplied ("x10") or divided ("o10") by 10 in the baseline solar composition.

\begin{tabular}{|c|c|c|c|c|c|c|c|c|c|c|c|c|c|c|c|c|}
\hline \multirow[b]{2}{*}{ Model } & \multicolumn{8}{|c|}{ Peak magnitude, $m$} & \multicolumn{8}{|c|}{ Peak epoch $t_{p}[\mathrm{~d}] /$ duration $\Delta t_{1 \mathrm{mag}}[\mathrm{d}]$} \\
\hline & $\mathrm{g}$ & $\mathrm{r}$ & $\mathrm{i}$ & $\mathrm{z}$ & y & $\mathrm{J}$ & $\mathrm{H}$ & $\mathrm{K}$ & $\mathrm{g}$ & $\mathrm{r}$ & $\mathrm{i}$ & $\mathrm{z}$ & $\mathrm{y}$ & $\mathrm{J}$ & $\mathrm{H}$ & $\mathrm{K}$ \\
\hline SolarUPm_baseline & -12.4 & -13.2 & -13.3 & -13.5 & -13.7 & -14.2 & -14.4 & -14.7 & $0.17 / 0.37$ & $0.23 / 0.62$ & $0.39 / 0.91$ & $0.56 / 1.12$ & $0.55 / 1.28$ & $0.80 / 1.77$ & $1.50 / 3.53$ & $1.64 / 3.83$ \\
\hline SolarUPm_br_o10 & -12.0 & -13.0 & -13.2 & -13.4 & -13.5 & -14.1 & -14.3 & -14.6 & $0.17 / 0.37$ & $0.22 / 0.59$ & $0.36 / 0.86$ & $0.52 / 1.05$ & $0.52 / 1.20$ & $0.75 / 1.70$ & $1.43 / 3.55$ & $1.64 / 3.90$ \\
\hline SolarUPm_br_x10 & -13.5 & -14.1 & -14.0 & -14.1 & -14.2 & -14.7 & -14.6 & -14.8 & $0.16 / 0.41$ & $0.19 / 0.73$ & $0.30 / 1.18$ & $0.74 / 1.42$ & $0.72 / 1.69$ & $0.83 / 2.09$ & $1.25 / 3.43$ & $1.51 / 3.59$ \\
\hline SolarUPm_ce_o10 & -12.4 & -13.2 & -13.4 & -13.6 & -13.7 & -14.2 & -14.4 & -14.7 & $0.17 / 0.43$ & $0.23 / 0.71$ & $0.44 / 0.95$ & $0.57 / 1.15$ & $0.53 / 1.30$ & $0.82 / 1.77$ & $1.49 / 3.50$ & $1.63 / 3.80$ \\
\hline SolarUPm_ce_x10 & -12.2 & -13.0 & -13.1 & -13.4 & -13.6 & -14.1 & -14.3 & -14.7 & $0.16 / 0.28$ & $0.19 / 0.41$ & $0.26 / 0.77$ & $0.48 / 1.01$ & $0.51 / 1.19$ & $0.77 / 1.76$ & $1.45 / 3.58$ & $1.65 / 3.88$ \\
\hline SolarUPm_dy_o10 & -12.4 & -13.2 & -13.4 & -13.6 & -13.7 & -14.2 & -14.4 & -14.7 & $0.17 / 0.37$ & $0.22 / 0.61$ & $0.37 / 0.92$ & $0.56 / 1.13$ & $0.55 / 1.28$ & $0.79 / 1.77$ & $1.50 / 3.54$ & $1.64 / 3.82$ \\
\hline SolarUPm_dy_x10 & -11.9 & -12.9 & -13.2 & -13.5 & -13.6 & -14.2 & -14.3 & -14.6 & $0.18 / 0.40$ & $0.23 / 0.64$ & $0.42 / 0.86$ & $0.56 / 1.03$ & $0.57 / 1.21$ & $0.79 / 1.68$ & $1.25 / 3.53$ & $1.55 / 3.91$ \\
\hline SolarUPm_er_o10 & -12.4 & -13.2 & -13.3 & -13.5 & -13.7 & -14.2 & -14.3 & -14.7 & $0.17 / 0.37$ & $0.22 / 0.62$ & $0.40 / 0.92$ & $0.56 / 1.13$ & $0.55 / 1.36$ & $0.81 / 1.88$ & $1.48 / 3.56$ & $1.63 / 3.81$ \\
\hline SolarUPm_er_x 10 & -12.2 & -13.1 & -13.3 & -13.5 & -13.6 & -14.1 & -14.4 & -14.7 & $0.17 / 0.37$ & $0.22 / 0.61$ & $0.40 / 0.89$ & $0.55 / 1.05$ & $0.52 / 1.06$ & $0.63 / 1.46$ & $1.32 / 3.36$ & $1.66 / 3.89$ \\
\hline SolarUPm_eu_o10 & -12.4 & -13.2 & -13.3 & -13.5 & -13.7 & -14.2 & -14.4 & -14.7 & $0.17 / 0.37$ & $0.22 / 0.62$ & $0.38 / 0.92$ & $0.56 / 1.12$ & $0.56 / 1.28$ & $0.80 / 1.77$ & $1.48 / 3.53$ & $1.64 / 3.82$ \\
\hline SolarUPm_eu_x10 & -12.3 & -13.2 & -13.3 & -13.5 & -13.6 & -14.2 & -14.4 & -14.7 & $0.17 / 0.35$ & $0.22 / 0.60$ & $0.37 / 0.91$ & $0.56 / 1.12$ & $0.57 / 1.31$ & $0.78 / 1.79$ & $1.48 / 3.55$ & $1.64 / 3.84$ \\
\hline SolarUPm_gd_o10 & -12.4 & -13.2 & -13.3 & -13.5 & -13.7 & -14.2 & -14.4 & -14.7 & $0.17 / 0.37$ & $0.22 / 0.62$ & $0.39 / 0.92$ & $0.56 / 1.12$ & $0.55 / 1.29$ & $0.80 / 1.81$ & $1.49 / 3.53$ & $1.64 / 3.82$ \\
\hline SolarUPm_gd_x10 & -12.3 & -13.2 & -13.3 & -13.5 & -13.6 & -14.2 & -14.4 & -14.7 & $0.17 / 0.36$ & $0.21 / 0.60$ & $0.40 / 0.89$ & $0.56 / 1.11$ & $0.52 / 1.16$ & $0.66 / 1.55$ & $1.42 / 3.51$ & $1.60 / 3.85$ \\
\hline SolarUPm_ho_o10 & -12.4 & -13.2 & -13.3 & -13.5 & -13.7 & -14.2 & -14.4 & -14.7 & $0.17 / 0.37$ & $0.22 / 0.61$ & $0.39 / 0.91$ & $0.56 / 1.13$ & $0.56 / 1.30$ & $0.80 / 1.77$ & $1.50 / 3.53$ & $1.64 / 3.82$ \\
\hline SolarUPm_ho_x10 & -12.3 & -13.2 & -13.3 & -13.5 & -13.7 & -14.2 & -14.4 & -14.7 & $0.17 / 0.37$ & $0.22 / 0.62$ & $0.40 / 0.91$ & $0.55 / 1.03$ & $0.55 / 1.18$ & $0.81 / 1.75$ & $1.48 / 3.51$ & $1.62 / 3.84$ \\
\hline SolarUPm_la_o10 & -12.4 & -13.2 & -13.3 & -13.5 & -13.7 & -14.2 & -14.4 & -14.7 & $0.18 / 0.37$ & $0.22 / 0.61$ & $0.40 / 0.92$ & $0.57 / 1.12$ & $0.55 / 1.28$ & $0.79 / 1.77$ & $1.49 / 3.53$ & $1.64 / 3.82$ \\
\hline SolarUPm_la_x10 & -12.3 & -13.2 & -13.3 & -13.5 & -13.7 & -14.2 & -14.4 & -14.7 & $0.17 / 0.36$ & $0.22 / 0.62$ & $0.37 / 0.87$ & $0.56 / 1.12$ & $0.54 / 1.29$ & $0.81 / 1.76$ & $1.47 / 3.52$ & $1.64 / 3.83$ \\
\hline SolarUPm_nd_o10 & -12.5 & -13.4 & -13.5 & -13.8 & -13.9 & -14.4 & -14.6 & -14.7 & $0.17 / 0.37$ & $0.22 / 0.63$ & $0.33 / 0.94$ & $0.58 / 1.19$ & $0.60 / 1.35$ & $0.83 / 1.74$ & $1.34 / 3.33$ & $1.59 / 3.91$ \\
\hline SolarUPm_nd_x10 & -11.5 & -12.5 & -12.7 & -12.8 & -13.0 & -13.5 & -13.9 & -14.4 & $0.18 / 0.39$ & $0.22 / 0.59$ & $0.34 / 0.77$ & $0.37 / 0.91$ & $0.40 / 1.09$ & $0.78 / 1.90$ & $1.49 / 4.03$ & $1.77 / 4.19$ \\
\hline SolarUPm_pd_o10 & -12.3 & -13.2 & -13.3 & -13.5 & -13.7 & -14.2 & -14.3 & -14.7 & $0.17 / 0.37$ & $0.22 / 0.61$ & $0.38 / 0.90$ & $0.55 / 1.11$ & $0.55 / 1.27$ & $0.78 / 1.76$ & $1.48 / 3.53$ & $1.64 / 3.84$ \\
\hline SolarUPm_pm_o10 & -12.4 & -13.3 & -13.4 & -13.6 & -13.7 & -14.2 & -14.4 & -14.7 & $0.17 / 0.37$ & $0.22 / 0.61$ & $0.37 / 0.91$ & $0.56 / 1.13$ & $0.53 / 1.29$ & $0.80 / 1.76$ & $1.49 / 3.51$ & $1.62 / 3.79$ \\
\hline SolarUPm_pm_x10 & -12.0 & -12.8 & -13.1 & -13.4 & -13.5 & -14.0 & -14.3 & -14.5 & $0.18 / 0.38$ & $0.23 / 0.65$ & $0.41 / 0.88$ & $0.55 / 1.08$ & $0.58 / 1.23$ & $0.78 / 1.84$ & $1.51 / 3.65$ & $1.74 / 4.04$ \\
\hline SolarUPm_pr_o10 & -12.4 & -13.2 & -13.4 & -13.7 & -13.8 & -14.3 & -14.4 & -14.7 & $0.17 / 0.38$ & $0.22 / 0.63$ & $0.40 / 0.94$ & $0.60 / 1.16$ & $0.65 / 1.32$ & $0.82 / 1.75$ & $1.55 / 3.42$ & $1.61 / 3.79$ \\
\hline SolarUPm_pr_x10 & -12.2 & -13.0 & -13.0 & -13.1 & -13.2 & -13.7 & -14.1 & -14.6 & $0.17 / 0.35$ & $0.22 / 0.58$ & $0.31 / 0.75$ & $0.39 / 0.93$ & $0.36 / 1.11$ & $0.72 / 1.86$ & $1.30 / 3.83$ & $1.65 / 3.98$ \\
\hline SolarUPm_se_o10 & -11.7 & -12.7 & -13.0 & -13.2 & -13.4 & -13.9 & -14.2 & -14.5 & $0.18 / 0.37$ & $0.22 / 0.56$ & $0.35 / 0.80$ & $0.48 / 0.98$ & $0.48 / 1.12$ & $0.70 / 1.65$ & $1.36 / 3.59$ & $1.65 / 3.99$ \\
\hline SolarUPm_se_x10 & -13.9 & -14.5 & -14.3 & -14.3 & -14.3 & -14.8 & -14.7 & -14.8 & $0.17 / 0.43$ & $0.20 / 0.74$ & $0.28 / 1.26$ & $0.77 / 1.51$ & $0.76 / 1.82$ & $0.77 / 2.17$ & $1.19 / 3.32$ & $1.41 / 3.44$ \\
\hline SolarUPm_sm_o10 & -12.6 & -13.2 & -13.4 & -13.6 & -13.7 & -14.2 & -14.4 & -14.7 & $0.17 / 0.36$ & $0.24 / 0.69$ & $0.45 / 0.97$ & $0.61 / 1.16$ & $0.56 / 1.35$ & $0.81 / 1.80$ & $1.51 / 3.50$ & $1.63 / 3.78$ \\
\hline SolarUPm_sm_x10 & -11.5 & -12.8 & -13.0 & -13.2 & -13.5 & -14.0 & -14.3 & -14.6 & $0.18 / 0.36$ & $0.19 / 0.45$ & $0.24 / 0.69$ & $0.37 / 0.90$ & $0.48 / 0.97$ & $0.71 / 1.63$ & $1.45 / 3.68$ & $1.76 / 4.05$ \\
\hline SolarUPm_tb_o10 & -12.4 & -13.2 & -13.3 & -13.5 & -13.7 & -14.2 & -14.4 & -14.7 & $0.17 / 0.37$ & $0.22 / 0.61$ & $0.39 / 0.91$ & $0.56 / 1.12$ & $0.55 / 1.28$ & $0.80 / 1.77$ & $1.50 / 3.54$ & $1.63 / 3.82$ \\
\hline SolarUPm_tb_x10 & -12.3 & -13.2 & -13.3 & -13.5 & -13.7 & -14.2 & -14.3 & -14.7 & $0.17 / 0.37$ & $0.22 / 0.61$ & $0.39 / 0.91$ & $0.56 / 1.12$ & $0.56 / 1.27$ & $0.79 / 1.71$ & $1.37 / 3.46$ & $1.65 / 3.87$ \\
\hline SolarUPm_te_o10 & -12.2 & -13.1 & -13.3 & -13.5 & -13.6 & -14.2 & -14.3 & -14.6 & $0.17 / 0.37$ & $0.22 / 0.60$ & $0.39 / 0.89$ & $0.54 / 1.09$ & $0.54 / 1.25$ & $0.78 / 1.74$ & $1.47 / 3.54$ & $1.64 / 3.85$ \\
\hline SolarUPm_te_x10 & -13.1 & -13.8 & -13.8 & -13.9 & -14.0 & -14.5 & -14.5 & -14.8 & $0.17 / 0.39$ & $0.21 / 0.69$ & $0.37 / 1.07$ & $0.67 / 1.30$ & $0.64 / 1.52$ & $0.85 / 1.96$ & $1.50 / 3.50$ & $1.61 / 3.70$ \\
\hline SolarUPm_tm_o10 & -12.4 & -13.2 & -13.3 & -13.5 & -13.7 & -14.2 & -14.4 & -14.7 & $0.17 / 0.37$ & $0.22 / 0.61$ & $0.38 / 0.92$ & $0.56 / 1.12$ & $0.53 / 1.29$ & $0.79 / 1.78$ & $1.54 / 3.62$ & $1.63 / 3.79$ \\
\hline SolarUPm_tm_x 10 & -12.3 & -13.2 & -13.3 & -13.5 & -13.7 & -14.2 & -14.3 & -14.7 & $0.17 / 0.37$ & $0.22 / 0.62$ & $0.39 / 0.91$ & $0.56 / 1.12$ & $0.56 / 1.26$ & $0.76 / 1.69$ & $1.20 / 3.22$ & $1.66 / 3.96$ \\
\hline SolarUPm_u_o10 & -13.3 & -13.8 & -13.6 & -13.7 & -13.8 & -14.3 & -14.4 & -14.7 & $0.16 / 0.32$ & $0.18 / 0.49$ & $0.22 / 0.87$ & $0.53 / 1.10$ & $0.51 / 1.25$ & $0.75 / 1.73$ & $1.45 / 3.50$ & $1.61 / 3.81$ \\
\hline SolarUPm_u_x10 & -11.0 & -12.3 & -13.0 & -13.3 & -13.4 & -14.1 & -14.4 & -14.7 & $0.19 / 0.52$ & $0.27 / 0.73$ & $0.46 / 0.87$ & $0.59 / 1.14$ & $0.66 / 1.34$ & $0.86 / 1.86$ & $1.53 / 3.59$ & $1.69 / 3.86$ \\
\hline SolarUPm_yb_o10 & -12.4 & -13.2 & -13.3 & -13.5 & -13.7 & -14.2 & -14.4 & -14.7 & $0.17 / 0.38$ & $0.22 / 0.61$ & $0.39 / 0.92$ & $0.56 / 1.13$ & $0.55 / 1.30$ & $0.85 / 1.96$ & $1.58 / 3.64$ & $1.87 / 3.97$ \\
\hline SolarUPm_yb_x10 & -12.1 & -13.2 & -13.3 & -13.5 & -13.7 & -14.1 & -14.1 & -14.5 & $0.16 / 0.35$ & $0.21 / 0.60$ & $0.36 / 0.87$ & $0.55 / 1.07$ & $0.56 / 1.23$ & $0.59 / 1.55$ & $1.01 / 3.24$ & $1.40 / 3.94$ \\
\hline SolarUPm_zr_o10 & -12.3 & -13.2 & -13.3 & -13.5 & -13.7 & -14.2 & -14.3 & -14.7 & $0.17 / 0.37$ & $0.22 / 0.64$ & $0.39 / 0.99$ & $0.56 / 1.19$ & $0.54 / 1.31$ & $0.78 / 1.77$ & $1.50 / 3.53$ & $1.63 / 3.72$ \\
\hline SolarUPm_zr_x10 & -12.6 & -13.4 & -13.5 & -13.6 & -13.8 & -14.3 & -14.5 & -14.6 & $0.17 / 0.37$ & $0.21 / 0.57$ & $0.38 / 0.85$ & $0.57 / 1.04$ & $0.58 / 1.27$ & $0.83 / 1.82$ & $1.49 / 3.52$ & $1.83 / 4.03$ \\
\hline
\end{tabular}

Table 4. Properties of light curves for solar r-process abundance, varying the overall lanthanide fraction. The naming convention is "SolarRP_lanth_xlem[1-9]", which implies the total lanthanide abundance is divided by $10^{1}-10^{9}$ in the baseline solar composition. The models with “_1ep1" and “_1ep2" correspond to $X_{\text {lanth }}=0.25$ and 0.75 in Figure 4.

\begin{tabular}{|c|c|c|c|c|c|c|c|c|c|c|c|c|c|c|c|c|}
\hline \multirow[b]{2}{*}{ Model } & \multicolumn{8}{|c|}{ Peak magnitude, $m$} & \multicolumn{8}{|c|}{ Peak epoch $t_{p}[\mathrm{~d}] /$ duration $\Delta t_{1 \mathrm{mag}}[\mathrm{d}]$} \\
\hline & g & $\mathrm{r}$ & $\mathrm{i}$ & $\mathrm{z}$ & $\mathrm{y}$ & $\mathrm{J}$ & $\mathrm{H}$ & $\mathrm{K}$ & $\mathrm{g}$ & $\mathrm{r}$ & $\mathrm{i}$ & $\mathrm{z}$ & $\mathrm{y}$ & $\mathrm{J}$ & $\mathrm{H}$ & $\mathrm{K}$ \\
\hline SolarRP_lanth_x1ep2 & -7.6 & -9.5 & -10.4 & -11.0 & -11.2 & -12.0 & -12.9 & -13.8 & $0.14 / 0.29$ & $0.16 / 0.34$ & $0.22 / 0.41$ & $0.26 / 0.55$ & $0.27 / 0.63$ & $0.43 / 1.71$ & $1.11 / 4.20$ & $2.28 / 5.21$ \\
\hline SolarRP_lanth_x1ep1 & -9.3 & -10.9 & -11.8 & -12.1 & -12.3 & -12.9 & -13.5 & -14.2 & $0.19 / 0.40$ & $0.24 / 0.49$ & $0.30 / 0.57$ & $0.36 / 0.71$ & $0.36 / 0.78$ & $0.51 / 1.57$ & $1.07 / 3.91$ & $1.85 / 4.55$ \\
\hline SolarRP_lanth_x1em1 & -14.3 & -14.9 & -14.7 & -14.4 & -14.5 & -15.0 & -14.8 & -14.7 & $0.17 / 0.44$ & $0.24 / 0.74$ & $0.30 / 1.14$ & $0.73 / 1.47$ & $0.37 / 1.78$ & $0.72 / 2.21$ & $1.08 / 2.94$ & $1.16 / 3.06$ \\
\hline SolarRP_lanth_x1em2 & -15.5 & -15.6 & -15.2 & -15.0 & -15.0 & -15.2 & -14.4 & -14.0 & $0.26 / 0.52$ & $0.37 / 0.83$ & $0.58 / 1.22$ & $0.62 / 1.37$ & $0.62 / 1.51$ & $0.82 / 2.23$ & $0.90 / 2.17$ & $0.97 / 2.22$ \\
\hline SolarRP_lanth_x1em3 & -15.6 & -15.4 & -15.0 & -14.8 & -14.7 & -14.8 & -13.6 & -12.9 & $0.30 / 0.69$ & $0.33 / 1.15$ & $0.33 / 1.49$ & $0.33 / 1.56$ & $0.36 / 1.53$ & $0.97 / 2.94$ & $1.05 / 1.53$ & $0.34 / 2.63$ \\
\hline SolarRP_lanth_x1em4 & -15.6 & -15.3 & -15.0 & -14.8 & -14.7 & -14.6 & -13.5 & -12.9 & $0.30 / 0.74$ & $0.33 / 1.26$ & $0.33 / 1.60$ & $0.34 / 0.72$ & $0.36 / 0.72$ & $1.00 / 1.43$ & $0.35 / 1.43$ & $0.34 / 0.75$ \\
\hline SolarRP_lanth_x1em5 & -15.6 & -15.3 & -15.0 & -14.8 & -14.7 & -14.6 & -13.5 & -12.9 & $0.30 / 0.74$ & $0.33 / 1.28$ & $0.33 / 1.61$ & $0.34 / 0.70$ & $0.36 / 0.70$ & $1.00 / 1.40$ & $0.35 / 1.42$ & $0.34 / 0.73$ \\
\hline SolarRP_lanth_x1em6 & -15.6 & -15.3 & -15.0 & -14.8 & -14.7 & -14.6 & -13.5 & -12.9 & $0.30 / 0.74$ & $0.32 / 1.28$ & $0.33 / 1.61$ & $0.34 / 0.70$ & $0.36 / 0.70$ & $1.00 / 1.40$ & $0.34 / 1.42$ & $0.34 / 0.73$ \\
\hline SolarRP_lanth_x1em7 & -15.6 & -15.3 & -15.0 & -14.8 & -14.7 & -14.6 & -13.5 & -12.9 & $0.30 / 0.74$ & $0.32 / 1.28$ & $0.33 / 1.61$ & $0.33 / 0.70$ & $0.36 / 0.70$ & $1.00 / 1.41$ & $0.34 / 1.42$ & $0.36 / 0.73$ \\
\hline SolarRP_lanth_x1em8 & -15.6 & -15.3 & -15.0 & -14.8 & -14.7 & -14.6 & -13.5 & -12.9 & $0.30 / 0.74$ & $0.32 / 1.28$ & $0.33 / 1.61$ & $0.34 / 0.70$ & $0.36 / 0.70$ & $1.00 / 1.40$ & $0.35 / 1.42$ & $0.35 / 0.73$ \\
\hline
\end{tabular}



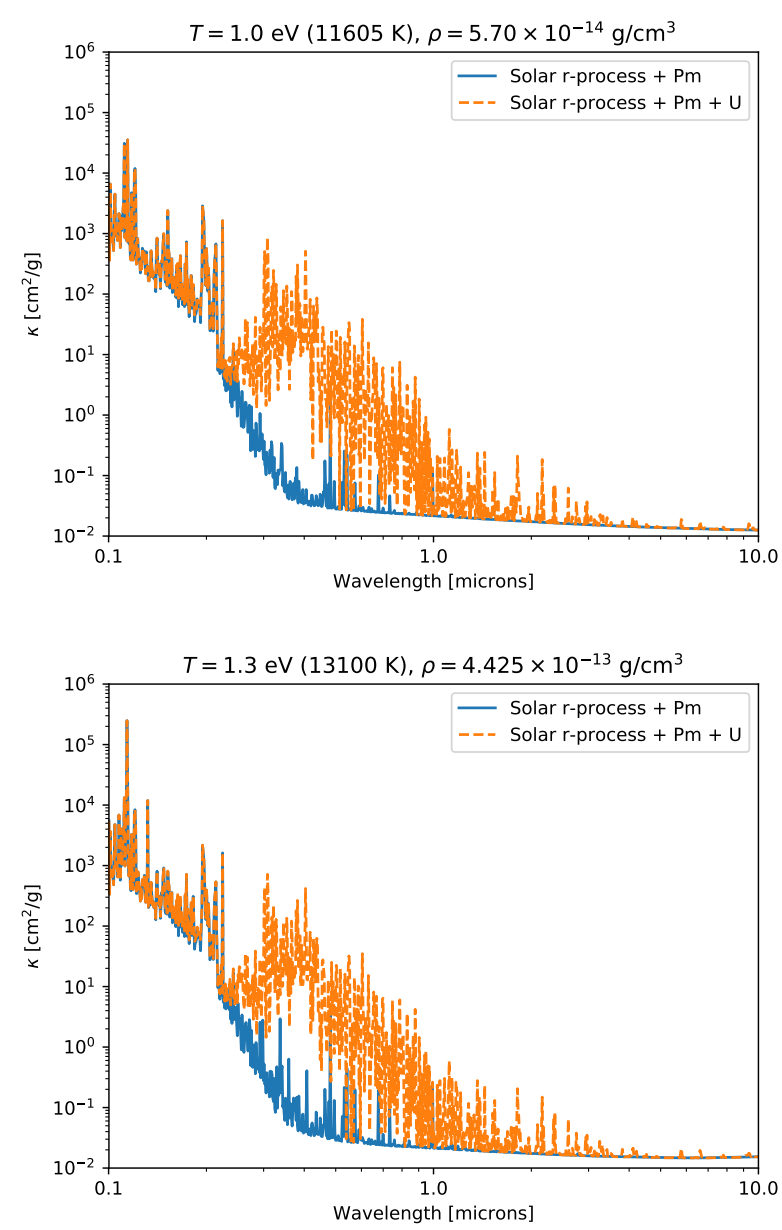

Figure 10. Opacity with and without uranium for two different temperature-density pairs. Top: $T=1.0 \mathrm{eV}, \rho=5.70 \times 10^{-14} \mathrm{~g} \mathrm{~cm}^{-3}$. Bottom: $T=1.3 \mathrm{eV}, \rho=4.425 \times 10^{-13} \mathrm{~g} \mathrm{~cm}^{-3}$. Above $T \sim 1.0 \mathrm{eV}$, the lines in uranium dramatically dominate the opacity from $0.2-$ $1 \mu \mathrm{m}$, obscuring emission in this wavelength range.
Surman, R., McLaughlin, G. C., Ruffert, M., Janka, H.-T., \& Hix, W. R. 2008, ApJL, 679, L117

Tanaka, M., Utsumi, Y., Mazzali, P. A., et al. 2017, PASJ, 69, 102

Tanvir, N. R., Levan, A. J., González-Fernández, C., et al. 2017, ApJL, 848, L27

Thielemann, K., F., Eichler, M., Panov, I. V., \& Wehmeyer, B. 2017, Annual Review of Nuclear and Particle Science, 67, annurev

Troja, E., Piro, L., van Eerten, H., et al. 2017, Nature, 551, 71

Vassh, N., Vogt, R., Surman, R., et al. 2018, arXiv e-prints, arXiv: 1810.08133

Vilén, M., Kelly, J., Kankainen, A., et al. 2018, Physical review letters, 120, 262701

Wollaeger, R. T., \& van Rossum, D. R. 2014, ApJS, 214, 28

Wollaeger, R. T., van Rossum, D. R., Graziani, C., et al. 2013, ApJS, 209, 36

Wollaeger, R. T., Korobkin, O., Fontes, C. J., et al. 2018, MNRAS, 478,3298

Zhu, Y., Wollaeger, R. T., Vassh, N., et al. 2018, ApJL, 863, L23 

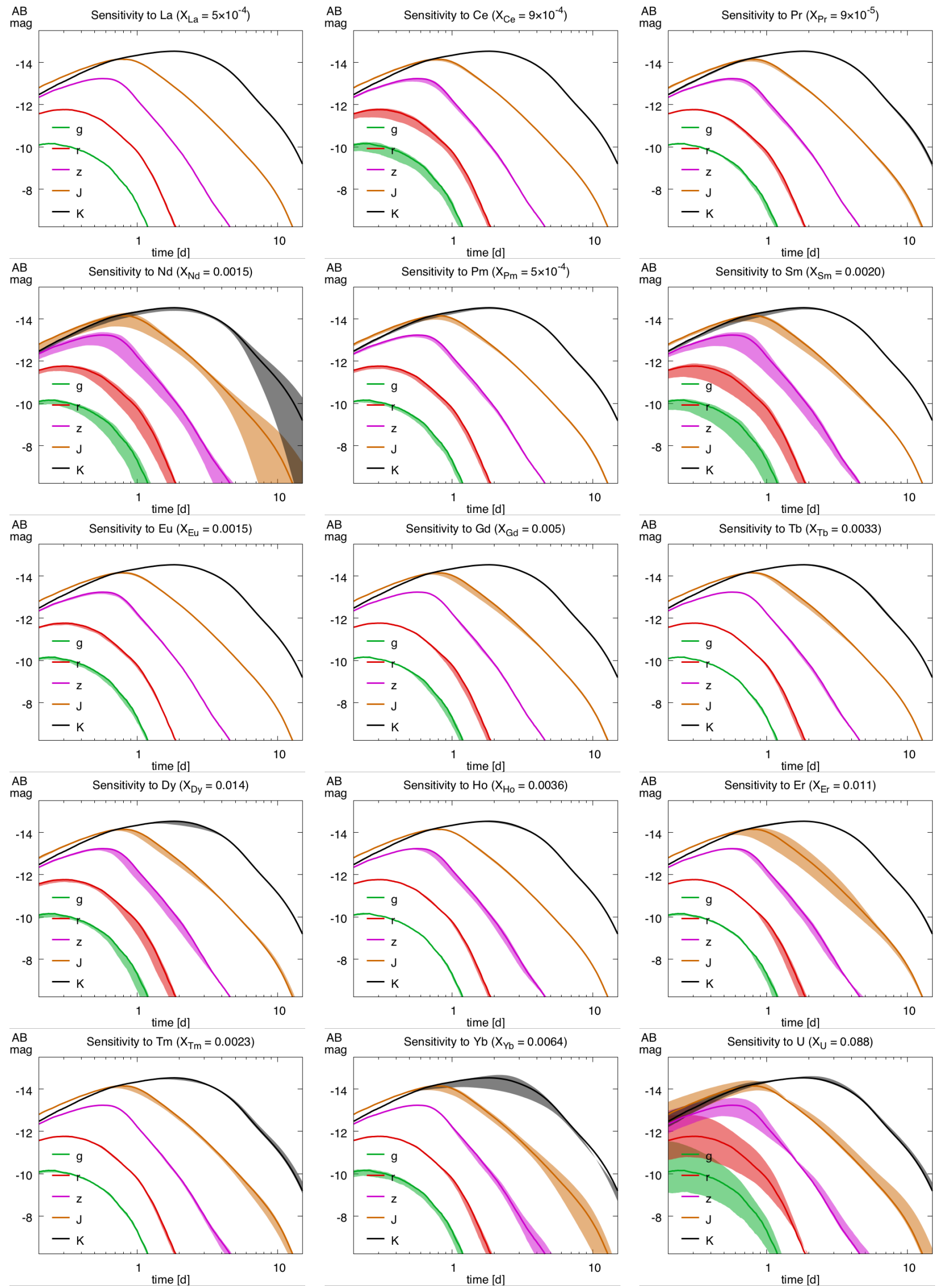

Figure 11. Sensitivity to the presence of individual lanthanides on the color band magnitudes of a kilonova with yields derived from dynamical ejecta calculations. 